\title{
Application des essais de pompage à l'évaluation du coefficient d'anisotropie hydraulique des sols aquifères
}

M. CASSAN

SA Fondasol Études BP 767

84035 Avignon Cedex
Nous avons précédemment présenté une méthode permettant d'évaluer le coefficient d'anisotropie des sols aquifères à partir d'essais Lefranc. Nous montrons maintenant que l'on peut, également de façon très simple, déterminer ce coefficient à partir des essais de pompage classiques dans le cas de puits imparfaits pourvu que l'or dispose d'au moins deux piézomètres.

Dans le cas d'un puits parfait, on pourra se ramener au cas du puits imparfait en arrêtant le forage du puits avant qu'il n'atteigne le substratum et en procédant à un essa de pompage spécifique, le forage du puits étant ensuite poursuivi jusqu'à sa profondeur définitive.

Nous précisons les procédures de réalisation de ces essais pour tous les cas pratiques que l'on peut rencontrer et nous donnons les formules et les tables numériques permettant l'interprétation de ces essais.
NDLE : Les discussions sur cet article sont acceptées dans un délai de trois mois après sa parution.
A method to deduce the anisotropic coefficlent of soil from Lefranc lests was previousiy presented

The folowing paper explains how determining this coefficient from classical pumping tests, for the case of an unperfect well. can be as easy as the previously described method provided that two piezometers are used. The solution for a perfect well is given by the solution for an unperfect well except that the wel drilling is stopped before the bedrock is reached. At this point. the well is tested with a specific pumping process before continuing drilling to the final depth.

The pumpind test procedures, for anv field applications, are detalled in this paper with formulae and numerical tables for their interpretation. 
La mesure du coefficient de perméabilité des sols homogènes et isotropes se fait, à I'heure actuelle, essentiellement à l'aide d'essais in situ, à savoir l'essai Lefranc et l'essaj de pompage, ces deux essais ayant fait l'objet d'une normalisation : normes Afnor NFP-94.132 pour le premier et NEP-94.130 pour le second.

Depuis plusieurs années, l'essai Lefranc tend à se généraliser au détriment de l'essai de pompage parce qu'il peut ètre considéré comme relativement rapide et qu'il est réalisable á toute profondeur au fur et à mesure de l'exécution d'un forage de recomnaissance usuel, et cela avec un matériel légrer, alors que l'essa: de pompage, qui nécessite un matériel lourd, est un essai beaucoup plus long et évidemment beaticoup plus cher.

Bien qu'ti semble, par conséquent, évident que les causes du développement croissant de l'utilisation de l'essai Lefranc soient de nature plus écononiques que techniques, il nous a paru que cet essai était susceptible de se prêter, moyennant une procédure appropriée, à une évaluation du coefficient d'anisotropie $\alpha$ défini. rappeions-le, comme le rapport du coefficient de perméabilité horizontale $k$, au coefficient de perméabilité verticale $k$. C'est pourquoi, nous avons cru utile d'étudier plus en détail ce problème et nos conclusions ont fait lobjet d'une précéciente communication [5]

Mais il ne faut pas perdre de vue que maloré les méthodes modernes d"interprétation de l'essai Lefranc qui permettent d'une part de déceler et d'éliminer les essais non représentatifs, et d'autre part d'exploiter valablement les essais partiellement perturbés par des phénomènes parasites, l'essai Lefranc reste un essai ponctuel avec les limites que cela implique alors que l'essai de pompage est un essai « totalisateur w qui rend compte de la perméabilité moyenne d'un volume de sol important.

C'est donc le seul essai qui puisse donner une idée relativement globale du comportement hydraulique du sol à l'échelle d'un projet et on ne peut imaginer qu'une étude sérieuse, où interviennent des rabattements et des débits d'exhaure ou de fuite importants, ptrisse être conduite sans y avoir recours. C'est pourquoi, afin d'accroitre le domaine d'application de cet essai indispensable qu'est l'essai de pompage et de le valoriser davantage si besoin était, il nous a paru important d'étudier les possibilités qu'il peut offrir dans la clètermination du coefficient d'anisotrople.

C'est cette étude que nous présentons dans la prèsente communication.

\section{Principe de la méthode}

Rappelons qu'un essai de pompage nécessite la réalisation d'un puits d'un diamétre relativement important par rapport à celui des forages de reconnaissance usuels (par exemple 0,50 à $1.00 \mathrm{~m}$ ) et la mise en place d'un certain nombre de piézomètres (au moins trois selon la norme NFP-94.130) situés à des distances croissantes du puits.

L'essai consiste alors à mesurer, en fonction du temps, l'évolution du niveau de l'eau dans le puits, mais surtout dans les piézomètres, et cela, si possible, jusqu'à l'obtention du régime permanent. Si ce régime n'est pas atteint, on peut néanmoins interpréter l'essai en régime transitoire, d'autant plus que, moyennant certaines approximations (approximation logarithmique de Jacob), on peut démontrer qu'à tout instant les formules du régime permanent restent applicables, le régime transitoire apparaissant alors comme une succession de régimes permanents.

L'interprétation classique de l'essai de pompage suppose que le sol étudié est homogène et isotrope, ce quil implique que l'équation de continuité soit une écuation de Laplace. Or, on sait que dans un milieu anjsotrope il n'en est plus ainsi.

Pour déterminer le coefficient d'anisotropie $\alpha$, il faut alors transformer, par une affinité géomètrique, le milieu anisotrope réel en un milieu isotrope fictif dans lequel l'équation de continuité est bien une équation de Laplace, et où le coefficient de perméabilité k satisfait à une autre condition arbitraire [5].

On choisit évidemment une condition qui simplifie le problème, et, dans le cas présent, nous avons adopté celle qui consiste à ne pas modifier les distances verticales.

La transformation est donc telle que :

$$
\begin{aligned}
& k=k_{,} \\
& x^{\prime}=\frac{x}{\sqrt{\alpha}} \\
& y^{\prime}=\frac{y}{\sqrt{\alpha}} \\
& z^{\prime}=z
\end{aligned}
$$

On démontre, par ailleurs, que le débit $Q$ dans le milieu réel est lié au débit $Q^{\prime}$ dans le milieu transformé par la relation :

$$
Q^{\prime}=\frac{Q}{\alpha}
$$

\section{2}

\section{Puits parfaits}

Rappelons qu'un puits est dit parfait lorsqu'il traverse entièrement la nappe aquifere et qu'il atteint le substratum étanche. C'est done un cas relativement rare mais qui présente l'avantage d'une approche théorique simple.

\section{2 至}

\section{Nappe en charge}

Dans ce cas, la formule de Dupuit donne l'équation de la méridienne de la surface piézométrique jusque et y compris dans le puits (Fig. 1).

Dans un piézomètre situé à la distance r de l'axe du puits, le rabattement est donc:

$$
\mathrm{h}=\frac{Q}{2 \pi k H} \ln \frac{R_{a}}{r}
$$

oú $\mathrm{R}_{\mathrm{a}}$ est le rayon d'action qui ne peut être déterminé qu'expérimentalement $[3,4]$.

En appliquant à cette relation la transformation définie par les équations [1], on obtient:

$$
r^{\prime}=\frac{r}{\sqrt{\alpha}} \quad z^{\prime}=z \quad Q^{\prime}=\frac{Q}{\alpha}
$$




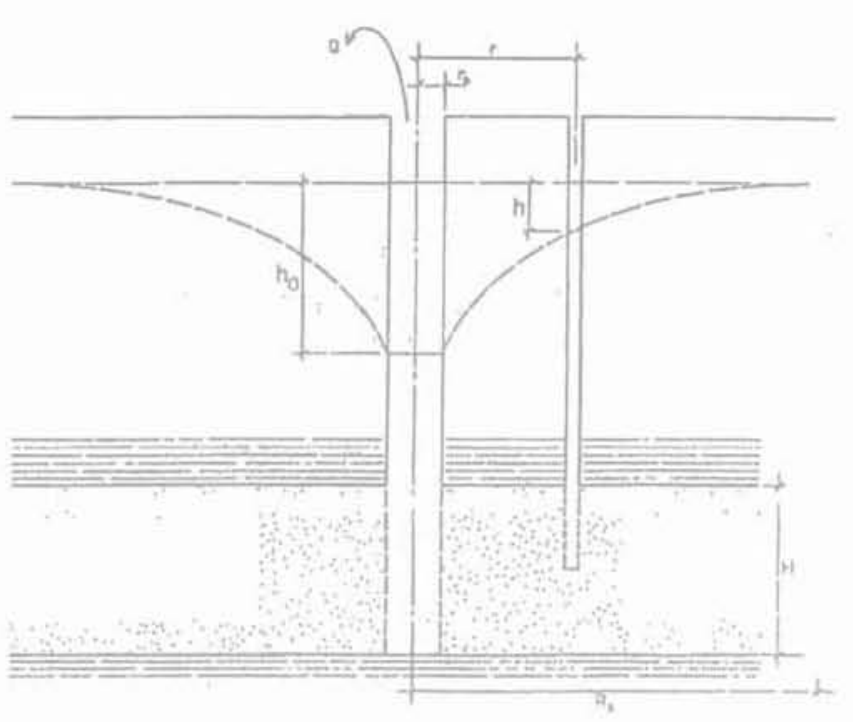

คG. 1 Puits parfait en nappe en charge. Perfect well in a confined aquifer

La transformée de l'équation (3) est alors:

$$
\mathrm{h}^{\prime}=\frac{Q^{\prime}}{2 \pi k_{1} H^{\prime}} \ln \frac{R_{2}^{\prime}}{r^{\prime}}
$$

Mais comme les distances verticales sont inchangées et que $\frac{R_{2}^{\prime}}{r^{\prime}}=\frac{R_{2}}{r}$, l'équation transformée devient tous calculs faits :

$$
\mathrm{h}=\frac{Q}{2 \pi k_{h} H} \ln \frac{R_{2}}{r}
$$

En comparant cette équation avec l'équation (3) on voit que le coefficient de perméabilité mesuré dans un essai de pompage selon les méthodes usuelles est le coefficient de perméabilité horizontale et quion ne peut done déterminer aînsi le coefficient danisotropie. $\square$ n'en est plus de même si l'on fait varier la fiche du puits dans l'aquifere, ce qui nous place dans le cas du puits imparfait que nous examinerons plus loin.

Toutefois, il est possible de résoudre le problème si on complète l'essai de pompage par de simples essais Lefranc normaux et si on applique la formule :

$$
\frac{k_{k}}{k}=\frac{m(\lambda) \sqrt{\alpha}}{m(\lambda \sqrt{\alpha})}
$$

oú $m(\lambda)$ et $m(\lambda \sqrt{a})$ sont les coefficients de forme des cavités Lefranc pour des élancements $\lambda$ (milieu réel) et $\lambda \sqrt{\alpha}$ (milieu transformé).

Puisque $k$, est donné par l'essai de pompage et $k$ par les essais Lefranc, l'équation précédente ne contient que la seule inconnue $\alpha$ et peut ètre résolue analytiquement dans certains cas et numériquement dans les autres [5].

Pratiquement, il faudra évidemment réaliser plusieurs essais Lefranc avec le même élancement pour obtenir une estimation moyenne de k sur toute l'épaisseur de l'aquifère, puisque, le puits étant par hypothèse parfait, l'estimation de $k_{h}$ est une estimation moyenne sur toute cette épaisseur.

\section{Nappe libre}

Dans le cas de la nappe libre, la formule de Dupuit ne donne pas le rabattement à la distance $\mathrm{r}$ du puits, mais la hauteur $H(r)$ de la surface libre au-dessus du substratum imperméable.

$$
\mathrm{H}_{0}^{2}-H^{2}=\frac{Q}{\pi k} \ln \frac{\mathrm{P}_{u}}{r}
$$

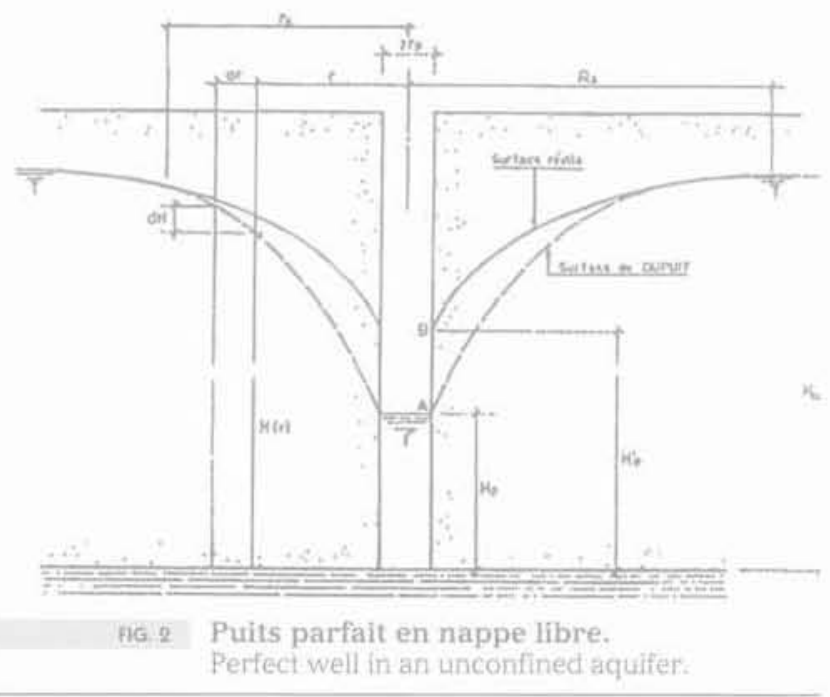

Malheureusement, cette formule ne correspond à l'équation de la méridienne de la surface de rabattement qu'au-clelà d'une valeur de r racine de l'équation :

$$
\frac{d H}{d r}=0,2
$$

Entre ce rayon critique et la surface du puits, on ne connait pas l'expression analytique de la méridienne qui ne rejoint d'ailleurs pas le niveau de l'eau dans le puits (hauteur de suintement). En revanche, on démontre $[3,7]$ que, dans le puits, la formule de Dupuit est exacte et a pour expression :

$$
H_{p}^{2}-H_{p}^{2}=\frac{Q}{\pi k} \ln \frac{R_{e}}{r_{p}}
$$

où $\mathrm{H}_{\text {}}$ et $\mathrm{r}_{\mathrm{n}}$ désignent respectivement la hauteur d'eau dans le puits et le rayon du puits.

Si on applique aux équations (5) et (6) la même transformation géométrique que précédemment, on obtient comme équations transformèes :

$H_{0}^{2}-H^{2}=\frac{Q}{\pi k_{h}} \ln \frac{R_{h}}{r}$ dans les piézomètres éloignès

et :

$H_{0}^{2}-H_{p}^{2}=\frac{Q}{\pi k_{h}} \ln \frac{R_{e}}{r_{p}}$ dans le puits.

On voit donc que le coefficient de perméabilité mesuré dans un essai de pompage en nappe libre est, lui aussi, égal au coefficient de perméabilité horizontale, et qu'il n'est possible d'évaluer le coefficient d'anisotropie qu'en réalisant le puits en au moins deux phases de longueur différente et avec pompage dans chacune d'elles avant d'atteindre le substratum, ou alors en effectuant, dans l'aquifère, des essais Lefranc normalisés. 


\section{Puits imparfaits}

Un puits imparfait est, rappelons-le, un puits qui ne descend pas jusqu'au substratum étanche.

\section{3a1}

\section{Nappe en charge}

Nous devons examiner les deux configurations suivantes:

- le substratum ètanche peut être considéré comme infiniment profond par rapport à la base du puits et, par conséquent, par rapport à l'éponte ètanche supérieure :

- le substratum étanche est situé à une distance finie de l'éponte supérieure et de la base du puits.

Dans un souci d'exhaustivité, nous examinerons également, bien qu'il soit contraire à la norme et par conséquent très rare, le cas d'un puits qui ne serait assorti d'aucun plézomètre.

\section{Substratum étanche infiniment profond}

\section{- Interprétation de type Lefranc en puits unique sans piézomètres}

La formule de Dupuit ne s'applique évidemment plus, mais on remarque que l'éponte supérieure étanche constitue une surface de courant. Il en résulte que les équipotentielles sont verticales au niveau de cette éponte.

Si on prend l'image du puits et du massif aquifère dans le miroir constitué par l'éponte supérieure (Fig. 3b) on voit que l'écoulement est identique à celui d'un essai Lefranc en milieu infini, et que le réseau d'écoulement autour du puits réel (Fig. 3a) est le même que le demi-réseau inférieur de la figure $3 \mathrm{~b}$.

Si D est la fiche du puits dans l'aquifère, on peut donc admettre que l'on pompe dans le puits réel le demi-débit que l'on pomperait dans la cavité fictive constituée par le puits et son image, pour une variation de charge sur les parois de la cavité égale au rabattement h dans le puits. $Q$ désignant le débit qui produit ce rabattement h, le débit nécessaire pour provoquer la mème variation de charge dans la cavité fictive de hauteur $2 \mathrm{D}$ sera donc $2 \mathrm{Q}$. Il en résulte, d'après la formule usuelle de l'essai Lefranc:

$$
2 Q=m_{0} k h B
$$
avec, puisque dans le cas général l'élancement $\lambda=\frac{2 \mathrm{D}}{\mathrm{B}}$
est supérieur à 1,5;

$$
\mathrm{m}_{\mathrm{o}}=\frac{2 \pi \lambda}{\operatorname{Argsh} \lambda}
$$

Le débit Q dans le puits s'exprime donc avec la formule usuelle de l'essai Lefranc, mais avec le coefficient de forme:

$$
\mathrm{m}=\frac{\pi \lambda}{\operatorname{Argsh} \lambda}
$$

On peut alors appliquer rigoureusement la méthode exposée pour l'essai Lefranc [5], en réalisant le forage du puits en deux phases, une première phase arrêtée à la profondeur $\mathrm{D}_{1}$ au-dessous de l'éponte étanche avec pompage sous un débit $Q_{1}$, provoquant un rabattement $\mathrm{h}$, dans le puits, et une deuxième phase correspondant à la profondeur définitive du puits $\mathrm{D}=\mathrm{D}_{2}$ avec un nouveau pompage sous débit $Q_{2}$ provoquant un rabattement $h$. On est alors ramené au problème traité dans la référence [5], c'est-à-dire à la résolution de l'équation :

$$
\mathrm{q}=\frac{\operatorname{Argsh} \mathrm{x}}{\operatorname{Argsh}(n \mathrm{x})}
$$

dans laquelle:

$$
\mathrm{q}=\frac{h_{1} Q_{2}}{n h_{2} Q_{1}} \quad \mathrm{n}=\frac{D_{2}}{D_{1}}=\frac{D}{D_{1}} \quad \mathrm{x}=\lambda_{1} \sqrt{\alpha}
$$

on en déduit :

$$
k_{h}=\frac{Q_{1}}{2 \pi \lambda_{h} h_{h} B} \operatorname{Argsh}\left(\lambda_{i} \sqrt{\alpha}\right)
$$

Si le régime permanent n'a pas èté atteint avec l'un ou avec les deux débits, on pourra évaluer, avec la méthode de la norme NFP-94.132, les valeurs asymptotiques des rabattements permanents $h_{1}$ et $h_{2}$ correspondant à chaque débit.

\section{- Interprétation à partir de piézomètres}

Considérons un piézomètre situé à la distance r de l'axe du puits et dont la prise de pression se situe à l'interface de l'aquifère et de l'éponte étanche (point P de la figure 4). Le niveau de l'eau dans ce piézomètre correspond donc à la charge hydraulique en P. Or, si on se réfère au schéma d'écoulement de la figure $3 \mathrm{~b}$, on voit que la charge en $\mathrm{P}$ n'est autre que le potentiel qui règne sur la surface équipotentielle passant par P, laquelle est un ellipsoỉde de révolution allongé (Fig.5).

D'après la théorie de l'essai Lefranc [3] cette surface équipotentielle et la cavité fictive de filtration de la figure 3b sont deux ellipsoïdes homofocaux de distance focale 2D. Or le débit qui s'écoule dans cette cavité fictive est le mème que celui qui traverse l'équipotentielle. Mais, comme nous l'avons vu pour le puits isolé, une variation de charge donnée h mesurée dans le piézomètre correspond à un débit à travers l'équipotentielle égal au double du débit réel Q pompé dans le puits.

Puisque toutes les surfaces équipotentielles ont même distance focale $2 \mathrm{D}$ que la cavité fictive, on peut donc écrire pour l'équipotentielle passant par P:

$$
2 \mathrm{Q}=\frac{2 \pi \lambda}{\operatorname{Argsh} \lambda} k h B
$$

ous : $\lambda=\frac{2 \mathrm{D}}{2 \mathrm{r}}=\frac{\mathrm{D}}{\mathrm{r}}$ et $\mathrm{B}=2 \mathrm{r}$.

La relation qui régit l'écoulement à la distance r du puits est donc finalement :

$$
\mathrm{Q}=\frac{\pi \lambda}{\operatorname{Argsh} \lambda} \mathrm{khB}
$$

En transformant commé précédemment le milieu réel anisotrope en un milleu réel isotrope sans modification des coordonnées verticales, la relation précédente devient :

$$
Q=\frac{\pi \lambda}{\operatorname{Argsh}(\lambda \sqrt{\alpha})} \cdot k_{h} h B
$$

Si on désigne par $\lambda_{0}$ l'élancement de la partie crèpinée du puits de diamètre $B_{i}=2 r_{0}$ et par $h_{n}$ le rabatte- 
(2)
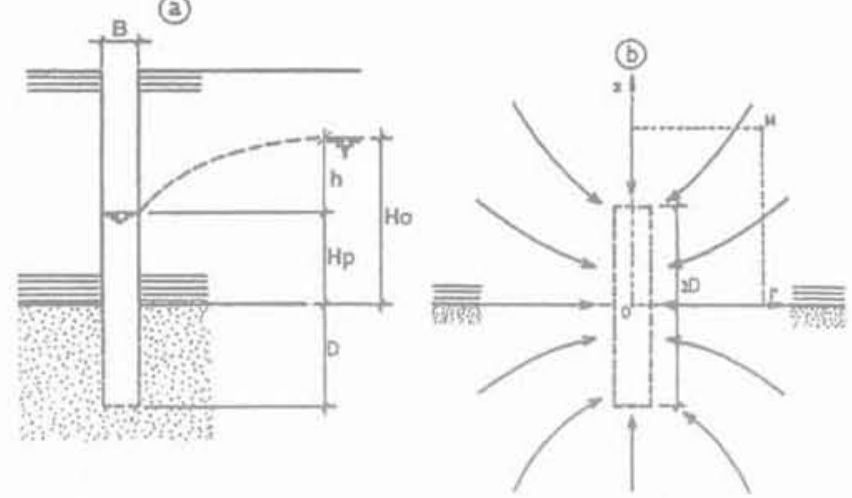

F10,3 Nappe en charge d'épaisseur infinie sans piézomètre.

Confined aquifer of infinite thickness without any piezometer.

ment dans le puits sous le débit $Q$, la formule précédente s'écrit, pour le puits :

$$
\mathrm{Q}=\frac{\pi \lambda_{0}}{\operatorname{Argsh}\left(\lambda_{e} \sqrt{\alpha}\right)} \cdot k_{h} h_{0} B_{0}
$$

avec: $\lambda_{0}=\frac{2 D}{B_{0}}$

Comme le débit est le même dans le puits et à travers l'équipotentielle, on déduit des relations (11) et (12):

$$
\frac{h}{h_{0}}=\frac{\operatorname{Argsh}(\lambda \sqrt{\alpha})}{\operatorname{Argsh}\left(\lambda_{0} \sqrt{\alpha}\right)}
$$

Si on pose :

$$
\begin{aligned}
& \lambda_{0}=n \lambda \rightarrow \quad \mathrm{n}=\frac{\mathrm{B}}{\mathrm{B}_{0}}>1 \\
& \mathrm{x}=\lambda \sqrt{\alpha}
\end{aligned}
$$

la relation précédente devient:

$$
\frac{h}{h_{0}}=\frac{\operatorname{Argsh} \mathrm{x}}{\operatorname{Argsh}(\mathrm{n} \mathrm{x})}
$$

C'est la même équation que l'équation (8) dans laquelle $q=\frac{h}{h}$.

Il est évidemment souhaitable de disposer de plusieurs piézomètres, ce qui permet d’avoir plusieurs évaluations de $\alpha$. Dans ce cas, la relation (13) peut s'appliquer à deux piézomètres dont les distances au puits seraient $r_{1}$ et $r_{2}$ avec $r_{2}>r_{1}$ et les variations de charge $h_{1}$ et $h_{2}$, avec évidemment $h_{2}<h_{1}$. On aurait alors:

$$
\frac{\mathrm{h}_{2}}{\mathrm{~h}_{1}}=\frac{\operatorname{Argsh} \mathrm{x}}{\operatorname{Argshn} \mathrm{x}}
$$

avec : $\mathrm{x}=\frac{D}{r_{2}} \sqrt{\alpha}=\lambda_{2} \sqrt{\alpha}$ et $\mathrm{n}=\frac{r_{2}}{r_{1}}$

Toutes choses restant égales par ailleurs, il est également possible de pomper avec plusieurs paliers de débits bien qu'un seul pompage soít suffisant.

On évalue le coeffícient de perméabilité horizontale directement à l'aide de la relation (11):

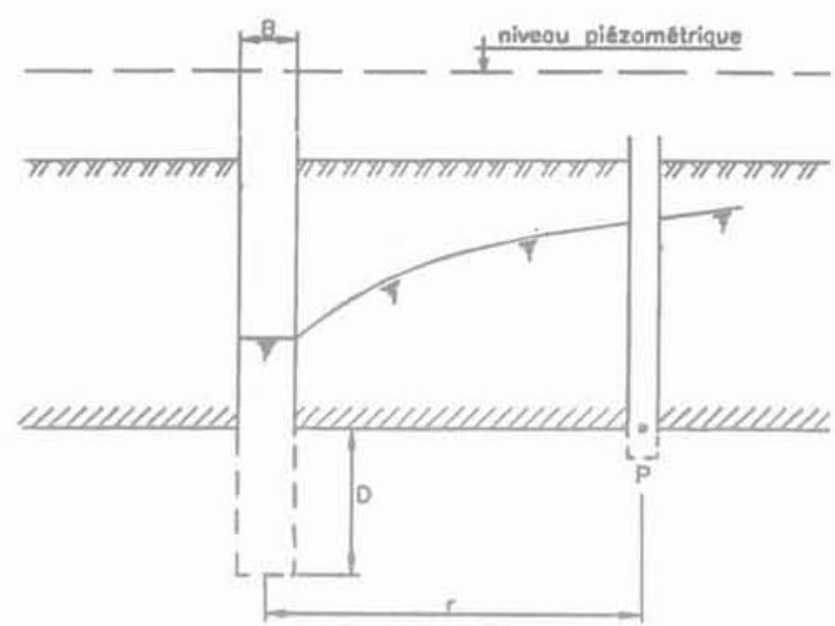

FiG.4 Nappe en charge d'épaisseur infinie avec piézomètre.

Confined aquifer of infinite thickness with piezometers.

$$
k_{h}=\frac{Q}{2 \pi h_{i} D} \operatorname{Argsh}\left(\lambda_{i} \sqrt{\alpha}\right)
$$

ou à partir de l'équation :

$$
\frac{k_{h}}{k_{i}}=\frac{\operatorname{Argsh}\left(\lambda_{i} \sqrt{\alpha}\right)}{\operatorname{Argsh} \lambda_{i}}
$$

avec :

$$
\mathrm{k}_{1}=\frac{\text { QArgsh } \lambda_{1}}{\pi \lambda_{i} h_{i} B_{i}}
$$

l'indice i prenant la valeur zéro sur le puits

$$
\lambda_{i}=\frac{D}{r_{i}}
$$

Comme précédemment, en régime transitoire, on pourra calculer $\mathrm{k}$ selon les prescriptions de la norme NFP-94.132, avec comme coefficient de forme:

$$
\mathrm{m}_{\mathrm{i}}=\frac{\pi \lambda_{i}}{\operatorname{Argsh} \lambda_{i}}
$$

L'intérêt de cette méthode avec piézomètres est qu'elle ne nécessite aucune variation de la hauteur crépinée du puits, c'est-à-dire aucun phasage dans le forage du puits, et qu'un pompage unique est théoriquement suffisant.

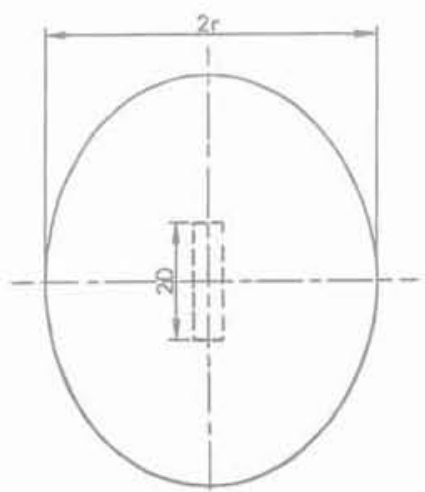

FIG.5 Équipotentielle homofocale à la cavité. Equipotential homofocal to the well. 


\section{$7 \sin$}

\section{Les deux horizons étanches sont à distance finie}

\section{- Puits unique sans piézomètres}

Soit H l'épaisseur de l'aquifère et D la fiche du puits dans cet aquifère, c'est-à-dire la fiche comptée, comme précédemment, à partir du niveau étanche supérieur (Fig. 6).

On peut, ici aussi, admettre que le réseau d'écoulement réel est constitué par la moitié inférieure du réseau d'écoulement autour d'une cavité de mème diamètre que le puits et de longueur 2D, et qui serait équidistante des deux niveaux ètanches d'une nappe en charge d'épaisseur $2 \mathrm{H}$. Ceci revient à considérer la disposition obtenue par symétrie de la configuration réelle par rapport à l'horizon étanche supérieur comme nous l'avons fait clans le cas précédent (Fig. 3b).

Le débit $Q$ qui provoque un rabattement $h$ dans le puits réel est toujours donné par la relation :

$$
\mathrm{Q}=\mathrm{mkhB}=\frac{1}{2} \mathrm{~m}_{1} \mathrm{khB}
$$

où $\mathrm{m}$, est le coefficient de forme de la cavité fictive de hauteur $2 \mathrm{D}$ dans le milieu image qui est alors un milieu à trois couches (Fig. 7).

Nous avons établi [2] que, dans un aquifère isotrope en charge de coefficient de perméabilité $k$, limité par deux couches horizontales de perméabilité $k_{1}$, le coefficient de forme pour une cavité équidistante des deux épontes, est donné par la relation.

$$
\frac{1}{m_{1}}=\frac{1}{m_{0}}+\frac{B}{4 \pi H} \ln \frac{\omega}{\omega-1}
$$

où: $\omega=\frac{k+k_{1}}{k-k_{1}}$

Pour le puits réel, on a donc:

$$
\frac{1}{m}=\frac{2}{m_{0}}+\frac{B}{2 \pi H} \ln \frac{\omega}{\omega-1}
$$

où $m$ est le coefficient de forme de la cavité de longueur 2D en milieu indéfini.

La formule (15) n'est théoriquement valable que lorsque T'épaisseur $\mathrm{H}$ de l'aquifère réel est telle que le demi-ellipsoïde homofocal à la cavité de filtration et tangent au substratum est assimilable à une sphère. On pourra admettre qu'il en est ainsi lorsque $\frac{D}{H}<0,3$. En effet, il est facile de montrer que le demi-petit axe r de l'ellipsoîde tangent au substratum est tel que

$$
\frac{r}{H}{\sqrt{1-\left(\frac{D}{H}\right)^{2}}}^{2}
$$

Pour $\frac{D}{H}=0,3$ on a en effet $\frac{r}{H}=0,95$ alors que le rayon $R$ de la sphère correspondante est tel que $\frac{R}{H}=1$.
L'écart entre r et $R$ est donc négligeable.

Lorsque les deux épontes qui limitent l'aquifère sont rigoureusement imperméables, c'est-à-dire lorsque $\mathrm{k}_{1}=0$, on a $\omega=1$ et $\ln \frac{\omega}{\omega-1}$ devient infini, ce qui est gênant.

Si on raisonne directement en supposant l'étanchéité absolue des épontes, on sait que l'on peut utiliser la théorie des images et considérer les deux horizons étanches qui limitent l'aquifère comme deux miroirs plans parallèles qui donnent une double infinité d'images.

On démontre alors [2] que le coefficient de forme pour le système tricouche devient:

$$
\frac{1}{m_{1}}=\frac{1}{m_{0}}+\frac{B}{4 \pi H}\left[\sum_{0}^{\infty} \frac{1}{2 n}+\sum_{0}^{\infty} \frac{1}{2 n-1}\right]
$$

où n est le nombre d'images, et, pour le système réel :

$$
\frac{1}{m}=\frac{2}{m_{0}}+\frac{B}{2 \pi H}\left[\sum_{1}^{\infty}\left(\frac{1}{2 n}+\frac{1}{2 n-1}\right)\right]
$$

La série harmonique $\sum_{1}^{x} \frac{1}{n}$ étant divergente ainsi que la série $\sum_{1}^{x} \frac{1}{2 n-1}\left(\right.$ puisque $\left.\frac{1}{2 n-1}>\frac{1}{2 n}\right)$, on retrouve bien, comme dans l'approche précédente, la divergence de $\frac{1}{m}$.

Cette divergence, bien qu'il s'agisse d'effets différents, constitue une difficulté théorique qui n'est pas sans rappeler, comme le faisait remarquer $\mathrm{H}$. Cambefort, celle que l'on rencontre avec le rayon d'action de la formule de Dupuit.

Mais nous faisons ici de la physique et non des mathématiques. On peut donc contourner cette difficulté en remarquant que dans deux terrains de perméabilité différente, dès que le coefficient de perméa-

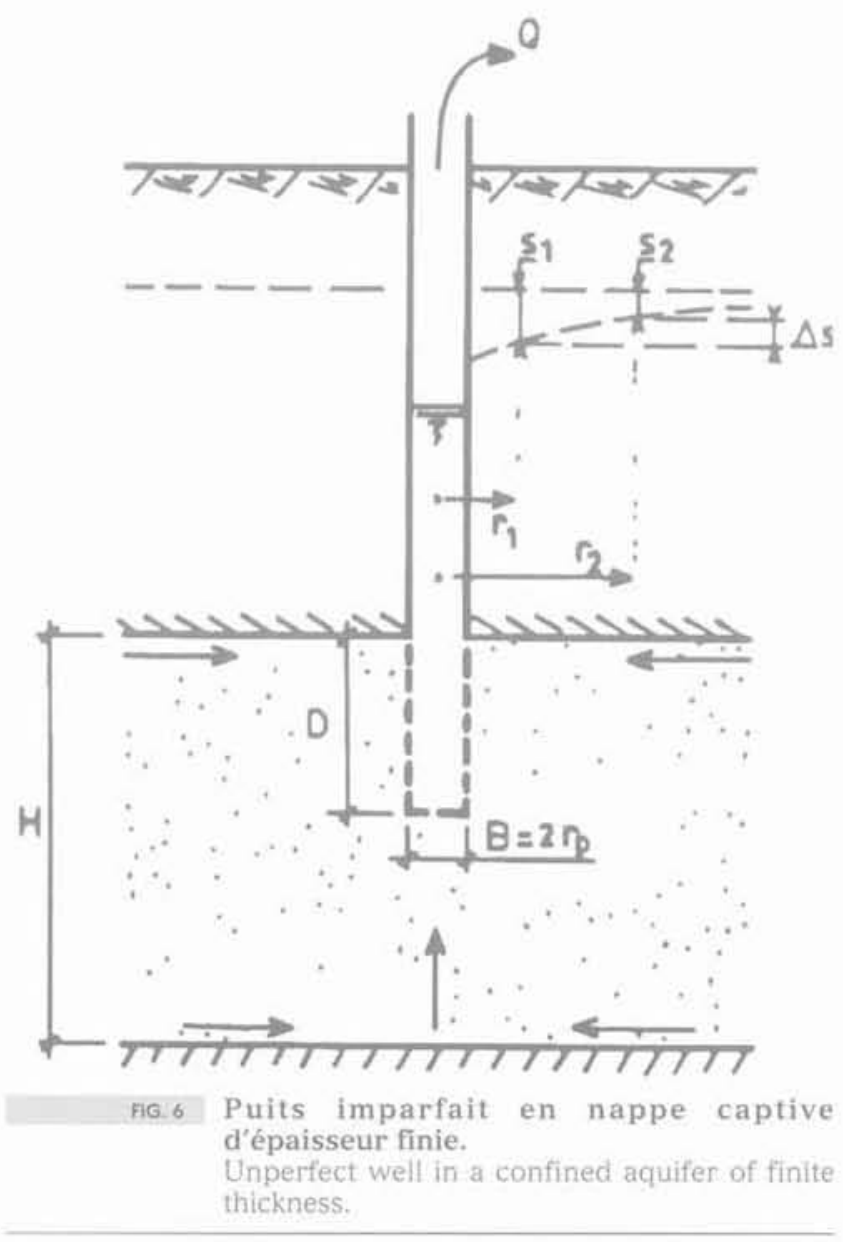


bilité de l'un de ces deux terrains diffère de celui de l'autre de deux puissances de 10, c'est-à-dire dans un rapport de 1 à 100 , on peut considérer le terrain le moins perméable cornme pratiquement étanche par rapport à l'autre.

Si nous voulons être plus prudents encore, on peut admettre que, dans le milieu tricouche considéré, la couche aquifere est mille fois plus perméable que les deux épontes, c'est-à-dire que :

$$
\frac{k_{1}}{k}=10^{-3}
$$

ou, ce qui revient au même, puisque $\frac{k_{1}}{k}$ est petit que :

$$
\omega=\frac{k+k_{1}}{k-k_{1}} \approx 1+2 \frac{k_{1}}{k}=1,002
$$

La relation (15) devient alors :

$$
\frac{1}{m}=\frac{2}{m_{0}}+\frac{6,22 B}{2 \pi H} \# \frac{2}{m_{0}}+\frac{B}{H}
$$

Dans la méthode des miroirs parallèles, cette expression correspond à un nombre d'images supérieur à 100, puisque pour 100 images, le coefficient de $\frac{B}{H}$ est de 0,93 . On peut donc admettre que l'expression proposée correspond à une étanchéité quasiment parfaite des deux épontes.

Le coefficient de forme est alors finalement :

$$
\mathrm{m}=\frac{m_{0} H}{2 H+m_{0} B}
$$

Le débit correspondant à un rabattement h dans le puits est alors:

$$
\mathrm{Q}=\frac{m_{0} H}{2 H+m_{0} B} k h B
$$

$$
\text { où : } m_{0}=\frac{4 \pi D}{B A r g s h\left(\frac{2 D}{B}\right)}
$$

Après quelques simplifications, on obtient:

$$
\mathrm{Q}=\frac{2 \pi}{2 \pi\left(\frac{D}{H}\right)+\operatorname{Argsh}\left(\frac{2 D}{B}\right)} \cdot k h D
$$

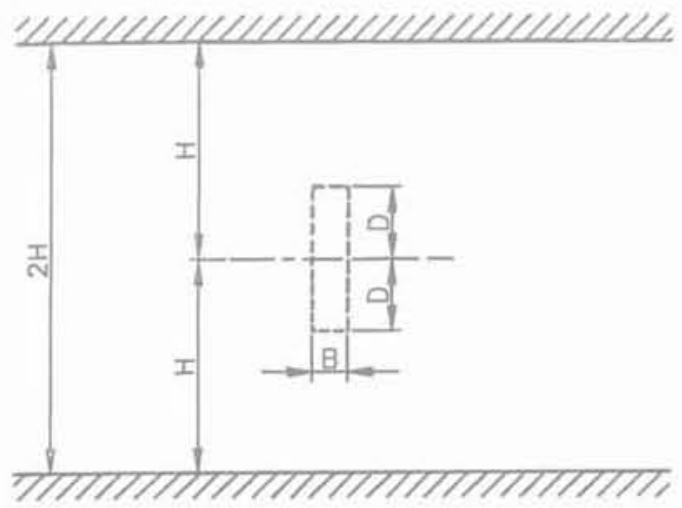

FiG.7 Système tricouche.

Three layer system.
Posons : $\frac{2 D}{B}=\lambda \quad$ et $\quad \frac{D}{H}=\mu$

il vient finalement :

$$
\mathrm{Q}=\frac{2 \pi}{2 \pi \mu+\operatorname{Argsh} \lambda} \cdot \mathrm{khD}
$$

La transformée de cette équation par l'affinité qui conserve les distances verticales conduit alors tous calculs faits à :

$$
Q=\frac{2 \pi}{2 \pi \mu+\operatorname{Argsh} \lambda \sqrt{\alpha}} k_{h} h D
$$

Pour évaluer $\alpha$, il suffit de réaliser deux essais de pompage, l'un avec le puits arrêté à la profondeur $D_{\text {}}$ sous l'éponte supérieure et le second avec le puits descendu à la cote définitive $\mathrm{D}_{2}$.

Dans le premier essai, on pompe avec un débit $Q_{1}$ et on obtient un rabattement $h$, et dans le deuxième essai on pompe un débit $Q_{2}$ qui produit un rabattement $h_{2}$.

On peut écrire :

$$
\begin{aligned}
& Q_{1}=\frac{2 \pi}{2 \pi \mu_{1}+\operatorname{Argsh} \lambda_{1} \sqrt{\alpha}} \cdot k_{h} h_{1} D_{1} \\
& Q_{2}=\frac{2 \pi}{2 \pi \mu_{2}+\operatorname{Argsh} \lambda_{2} \sqrt{\alpha}} \cdot k_{h} h_{2} D_{2}
\end{aligned}
$$

d'où : $\frac{h_{1} D_{1} Q_{2}}{h_{2} D_{2} Q_{1}}=\frac{2 \pi \mu_{1}+\operatorname{Argsh}\left(\lambda_{1} \sqrt{\alpha}\right)}{2 \pi \mu_{2}+\operatorname{Argsh}\left(\lambda_{2} \sqrt{\alpha}\right)}$

Posons :

$$
\frac{D_{2}}{D_{1}}=\mathrm{n} \quad \mathrm{x}=\lambda_{1} \sqrt{\alpha} \quad \mathrm{q}=\frac{h_{1} D_{1} Q_{2}}{h_{2} D_{2} Q_{1}}=\frac{h_{1} Q_{2}}{n h_{2} Q_{1}}
$$

L'équation précédente devient:

$$
q=\frac{2 \pi \mu_{1}+\operatorname{Argsh} x}{2 \pi n \mu_{1}+\operatorname{Argshn} x}
$$

Le premier membre de cette équation est connt expérimentalement et le paramètre $\mu_{1}$ est l'une des données géométriques. La résolution numérique de cette équation conduit à une valeur de $\mathrm{x}$ dont on décuit $\alpha$ puisque $\lambda_{1}$ est également connu.

On vérifie que lorsque $H$ tend vers linfini, c'est-à-dire lorsque le systeme tricouche tend vers le massif infini, $\mu$ tend vers zéro et on retrouve bien l'équation (8).

Connaissant $\alpha$, l'équation (17) donne directement:

$$
k_{h}=\frac{Q_{i}}{2 \pi h_{i} D_{i}}\left[2 \pi \mu_{j}+\operatorname{Argsh}\left(\lambda_{i} \sqrt{\alpha}\right)\right]
$$

Là aussi, en régime transitoire, on pourra évaluer $\mathrm{k}$ selon les prescriptions de la norme NFP-94132 avec comme coefficient de forme :

$$
\mathrm{m}_{i}=\frac{\pi \lambda_{i}}{2 \pi \mu_{i}+\operatorname{Argsh} \lambda_{i}}
$$

et calculer les valeurs de $h_{1}$ correspondant au régime permanent.

On remarque qu'en faisant $\mu_{i}=0$ dans l'équation (19) on retrouve bien l'équation (9). 


\section{- Puits avec piézomètres}

Cas où $\mu=\frac{\mathrm{D}}{\mathrm{H}}<0,3$

Dans ce cas, le puits est descendu directement à sa profondeur définitive et deux piézomètres suffisent alors pour résoudre le problème, à condition qu'ils soient disposés à une distance du puits inférieure à $0,95 \mathrm{H}$, condition en général facile à satisfaire pour un puits imparfait car ce type de puits ne se rencontre pratiquement que lorsque H est important.

On remarquera que la relation (17), que nous avons établie pour le puits, peut se généraliser pour un piézomètre situé à la distance $\mathrm{r}$ du puits. Il suffit pour cela de remplacer dans cette relation $\lambda=\frac{2 D}{B}$, par $\lambda=\frac{2 D}{2 r}=\frac{D}{T}$, le paramètre $\mu=\frac{D}{H}$, restant constant puisque $\mathrm{D}$ est une donnèe fixe.

Considérons alors deux piézomètres distants de $r_{1}$ et $r_{2}$ de l'axe du puits, avec par exemple $r_{2}>r_{1}$. Les rabattements $h_{1}$ et $h_{2}$ obtenus dans les piézomètres pour un débit $Q$ pompé dans le puits sont alors tels que $h_{1}>h_{2}$.

On a donc d'après (17) :

$$
Q=\frac{2 \pi}{2 \pi \mu+\operatorname{Argsh}\left(\lambda_{1} \sqrt{\alpha}\right)} k_{h} h_{1} D=\frac{2 \pi}{2 \pi \mu+\operatorname{Argsh}\left(\lambda_{2} \sqrt{\alpha}\right)} k_{\phi} h_{2} D
$$

d'où :

$$
\frac{h_{2}}{h_{1}}=\frac{2 \pi \mu+\operatorname{Argsh}\left(\lambda_{2} \sqrt{\alpha}\right)}{2 \pi \mu+\operatorname{Argsh}\left(\lambda_{1} \sqrt{\alpha}\right)}
$$

Posons : $\mathrm{n}=\frac{r_{2}}{r_{1}}>1$ c'est-à-dire $\lambda_{1}=\mathrm{n} \lambda_{2}$

$$
x=\lambda_{2} \sqrt{\alpha}
$$

La relation (20) s'écrit alors :

$$
\frac{h_{i}}{h_{1}}=\frac{2 \pi \mu+\operatorname{Argsh} x}{2 \pi \mu+\operatorname{Argsh}(n x)}
$$

प suffit donc de résoudre cette équation numériquement, ce qui ne présente pas de difficulté, et d'en déduire:

$$
\alpha=\left(\frac{x}{\lambda_{2}}\right)^{2}
$$

Connaissant $\alpha$ et en comparant les équations (16) et (17), on déduit :

$$
\frac{k_{h}}{k_{i}}=\frac{2 \pi \mu+\operatorname{Argsh}(\lambda, \sqrt{\alpha})}{2 \pi \mu+\operatorname{Argsh} \lambda_{i}}
$$

avec: $k_{1}=\frac{Q\left[2 \pi \mu+\operatorname{Argsh} \lambda_{i}\right]}{2 \pi D h_{i}}$

On peut aussi évaluer directement le coefficient de perméabilité horizontale :

$$
k_{h}=\frac{Q}{2 \pi \lambda_{i} D}\left[2 \pi \mu+\operatorname{Argsh}\left(\lambda_{i} \sqrt{\alpha}\right)\right]
$$

$$
\text { Cas où } \mu=\frac{\mathrm{D}}{\mathrm{H}}>0,3
$$

Pour évaluer $\alpha$, il faut mettre en place trois piézomètres dont les prises de pression doivent, comme précédemment, être voisines de l'éponte supérieure, et on utilisera la relation de Muskat et Nasberg [4] qui donne le rabattement $h$ dans un piézomètre situé à la distance $r$ de l'axe du puits :

$$
\mathrm{h}(\mathrm{r})=\frac{Q}{2 \pi k H}\left[\ln \frac{R_{0}}{r}+\Phi\left(R_{\mathrm{a}}\right)-\Phi(r)\right]
$$

avec $: \mathrm{R}_{\mathrm{a}}=$ rayon d'action du puits

$$
\Phi(r)=\frac{H}{D} \operatorname{Argsh} \frac{D}{r}-\operatorname{Argsh} \frac{H}{r}
$$

Transformons l'équation (22) par l'affinité qui conserve les distances verticales. On obtient alors :

$$
\mathrm{h}=\frac{Q}{2 \pi k_{h} H}\left[\ln \frac{R_{\mathrm{a}}}{r}+\Phi\left(\frac{R_{\mathrm{e}}}{\sqrt{\alpha}}\right)-\Phi\left(\frac{r}{\sqrt{\alpha}}\right)\right]
$$

Soient $r_{1}, r_{2}$ et $r_{3}$, les distances des piézomètres à l'axe du puits et $h_{1}$, $h_{2}$ et $h_{3}$, les rabattements qui y sont mesurés.

L'application de la formule (24) à chacun de ces piézomètres permet d'écrire:

$$
\frac{h_{1}-h_{2}}{h_{1}-h_{3}}=\frac{\ln \frac{r_{2}}{r_{1}}+\Phi\left(\frac{r_{2}}{\sqrt{\alpha}}\right)-\Phi\left(\frac{r_{1}}{\sqrt{\alpha}}\right)}{\ln \frac{r_{3}}{r_{1}}+\Phi\left(\frac{r_{3}}{\sqrt{\alpha}}\right)-\Phi\left(\frac{r_{1}}{\sqrt{\alpha}}\right)}
$$

Dans cette relation, les paramètres $h$ et $r_{1}$ sont connus. On se trouve donc en présence d'une équation à une inconnue $\alpha$, que l'on peut résoudre numériquement:

On peut également reprendre le calcul de $\alpha$ pour d'autres combinaisons possibles des $h_{\text {, }}$ et $r_{1}$, ce qui donne trois évaluations de $\alpha$ qui doivent être équivalentes.

Des équations (22) et (24), on déduit :

$$
\frac{k_{\mathrm{h}}}{\mathrm{k}}=\frac{\ln \frac{r_{2}}{r_{1}}+\Phi\left(\frac{r_{2}}{\sqrt{\alpha}}\right)-\Phi\left(\frac{r_{1}}{\sqrt{\alpha}}\right)}{\ln \frac{r_{3}}{r_{1}}+\Phi\left(r_{2}\right)-\Phi\left(r_{1}\right)}
$$

avec :

$$
\begin{aligned}
& \mathrm{k}=\frac{Q}{2 \pi\left(h_{1}-h_{2}\right) H}\left[\ln \frac{r_{2}}{r_{1}}+\Phi\left(r_{2}\right)-\Phi\left(r_{1}\right)\right]= \\
& \frac{Q}{2 \pi\left(h_{2}-h_{3}\right) H}\left[\ln \frac{r_{1}}{r_{2}}+\Phi\left(r_{3}\right)-\Phi\left(r_{2}\right)\right]=\text { etc. }
\end{aligned}
$$

ou directement :

$$
\begin{aligned}
& \mathrm{k}_{\mathrm{h}}=\frac{Q}{2 \pi\left(h_{1}-h_{2}\right) H}\left[\ln \frac{r_{2}}{r_{1}}+\Phi\left(\frac{r_{2}}{\sqrt{\alpha}}\right)-\Phi\left(\frac{r_{1}}{\sqrt{\alpha}}\right)\right]= \\
& \frac{Q}{2 \pi\left(h_{2}-h_{3}\right) H}\left[\ln \frac{r_{3}}{r_{2}}+\Phi\left(\frac{r_{3}}{\sqrt{\alpha}}\right)-\Phi\left(\frac{r_{2}}{\sqrt{\alpha}}\right)\right]=\text { etc. }
\end{aligned}
$$

\section{2}

\section{Nappe libre}

Le cas du puits imparfait dans une nappe libre n'a pas été résolu de façon théorique rigoureuse. Certains auteurs ont donné des formules semi-empiriques pour le rabattement dans le puits, mais non dans les piézomètres. 
Toutefois, d'après Schneebeli [8], on peut conserver les formules de la nappe en charge en remplaçant le rabattement $h(r)=H_{0}-H$ à la distance $r$ de l'axe du puits par l'expression:

$$
\mathrm{h}^{*}(\mathrm{r})=\frac{H_{0}^{2}-H^{2}}{2 H_{o}}
$$

soit encore :

$$
h^{*}=h\left(1-\frac{h}{2 H_{0}}\right)
$$

On voit que si h est petit devant $\mathrm{H}_{0}$ (et c'est souvent le cas pour un puits imparfait), on a :

$h^{*} \approx h$ et les formules de la nappe en charge sont alors parfaitement justifiées.

\section{7}

\section{Substratum étanche infiniment profond}

On se placera dans 1 hyypothèse oủ h est petit et on considérera le système constitué par le dispositif réel et par son symétrique par rapport au niveau initial de la nappe.

On se trouve donc comme dans le cas de la nappe en charge, en présence d'une cavité allongée de diamètre $B$ et de hauteur 2D aménagée dans un massif indéfini, $\mathrm{D}$ désignant la fiche du puits comptée à partir du niveau de la nappe, et on retrouve ainsi la configuration de l'essai Lefranc en milieu indéfini.

On pourrait alors évaluer le coefficient d'anisotropie en appliquant la méthode que nous avons développée précédemment dans le cas de la nappe captive avec substratum étanche infiniment profond, malgré l'existence d'une hauteur de suintement qui, dans le cas d'un puits imparfait en milieu semi-infini (disposition réelle), est plus faible que dans le cas du puits parfait. De plus, d'après notre hypothèse initiale relative aux faibles valeurs des rabattements, l'erreur que l'on peut faire en assimilant le rabattement dans le puits à la charge hydraulique n'est peut-être pas très significative.

Nous ne reviendrons pas sur cette méthode qui consiste à réaliser le forage du puits en plusieurs phases, avec pompage dans chaque phase, car il nous semble préférable d'utiliser la méthode avec piézomètres que nous avons développée ci-dessus pour la nappe en charge.

Il faudra alors prévoir au moins deux piézomètres (mais trois seraient souhaitables) disposés de façon à obtenir des rabattements susceptibles d'être considérés comme faibles par rapport à l'épaisseur de l'aquifère mais suffisamment contrastés pour conduire à des résultats fiables. La disposition de ces piézomètres pourra être déterminée en se fixant une valeur de l'épaisseur de l'aquifère très approximative, mais prudente, d'après ce que l'on connait de la géologie du site et en évaluant grossièrement un coefficient global de perméabilité en fonction de la nature des matériaux (formule de Hazen par exemple).

En considérant là aussi le symétrique du dispositif réel par rapport à la surface libre de la nappe au repos, on est ramené au cas de la nappe en charge et on est en droit d'utiliser les formules (13) et (13 bis).

Si on considère deux piézomètres dont les distances à l'axe du puits sont respectivement $r_{1}$ et $r_{2}$, avec par exemple $r_{2}>r_{1}$ et si $h_{1}$ et $h_{2}$ sont les rabattements correspondants (dans ce cas $h_{1}>h_{2}$ ), l'équation 13 bis que nous rappelons ci-après, est applicable :

$$
\frac{h_{2}}{h_{1}}=\frac{\operatorname{Argsh} x}{\operatorname{Argshn} x}
$$

avec : $\mathrm{x}=\frac{D}{r_{2}} \sqrt{\alpha}$ et $\mathrm{n}=\frac{r_{2}}{r_{1}}>1$.

Connaissant $\alpha$, on aura, d'après la relation (14), quel que soit le piézomètre :

$$
\frac{k_{h}}{k_{i}}=\frac{\operatorname{Argsh} \frac{D}{r_{i}} \sqrt{\alpha}}{\operatorname{Argsh} \frac{D}{r_{i}}}
$$

où $\mathrm{k}$ est évalué à l'aide de la formule (10). Mais on peut aussi évaluer directement $\mathrm{k}_{\mathrm{h}}$ à partir de la relation (11):

$$
\mathrm{k}_{\mathrm{h}}=\frac{Q}{2 \pi h_{i} D} \operatorname{Argsh}\left(\frac{D}{r_{1}} \sqrt{\alpha}\right)
$$

Si on dispose de plus de deux piézomètres, la formule (13 bis) pourra être utilisée plusieurs fois en permutant circulairement les indices de $h_{j}$ et $\mathrm{r}_{\mathrm{l}}$.

\section{3,062}

\section{Substratum étanche à distance finie}

Lorsque le substratum étanche se trouve à une distance finie $\mathrm{H}$ du niveau statique de la nappe (Fig. 9) on pourra évaluer le coefficient d'anisotropie à partir du rabattement dans le puits de deux manières différentes, soit en utilisant la formulation directe de Todd [4] soit en prenant le symétrique de la configuration réelle par rapport au niveau statique de la nappe et en utilisant la méthode développée pour la nappe en charge.

Rappelons que ces deux méthodes ne peuvent être utilisées que si le rabattement dans le puits $\mathrm{h}=\mathrm{H}_{\mathrm{o}}-\mathrm{H}_{\mathrm{b}}$ est négligeable par rapport à $\mathrm{H}_{\text {。 }}$

\section{- Utilisation de la formule de Todd dans le puits}

Cette formule est la suivante :

$$
\mathrm{h}=\frac{\mathrm{Q}}{2 \pi k D}\left[\frac{D}{10 H_{0}}+\ln \frac{\pi D}{B}\right]
$$

Si on effectue, comme dans tous les cas précédents, la transformation affine qui conserve les distances verticales, on obtient:

$$
\mathrm{h}=\frac{Q}{2 \pi k_{h} D}\left[\frac{D}{10 H_{0}}+\ln \frac{\pi D}{B} \sqrt{\alpha}\right]
$$

En réalisant deux essais avec deux valeurs différentes de $\mathrm{D}$, on obtient :

$$
\frac{D_{1} h_{1} Q_{2}}{D_{2} h_{2} Q 1}=\frac{\frac{D_{1}}{10 H_{0}}+\ln \frac{\pi D_{1}}{B} \sqrt{\alpha}}{\frac{D_{2}}{10 H_{0}}+\ln \frac{\pi D_{2}}{B} \sqrt{\alpha}}
$$

soit en posant :

$$
\mathrm{q}=\frac{D_{1} h_{1} Q_{2}}{D_{2} h_{2} Q_{1}} \quad \delta_{1}=\frac{D_{1}}{10 H_{0}} \quad \delta_{2}=\frac{D_{2}}{10 H_{o}} \quad \mathrm{n}=\frac{D_{2}}{D_{1}}=\frac{\delta_{2}}{\delta_{1}}
$$

et :

$$
\mathrm{x}=\frac{\pi D_{1}}{B} \sqrt{\alpha}
$$




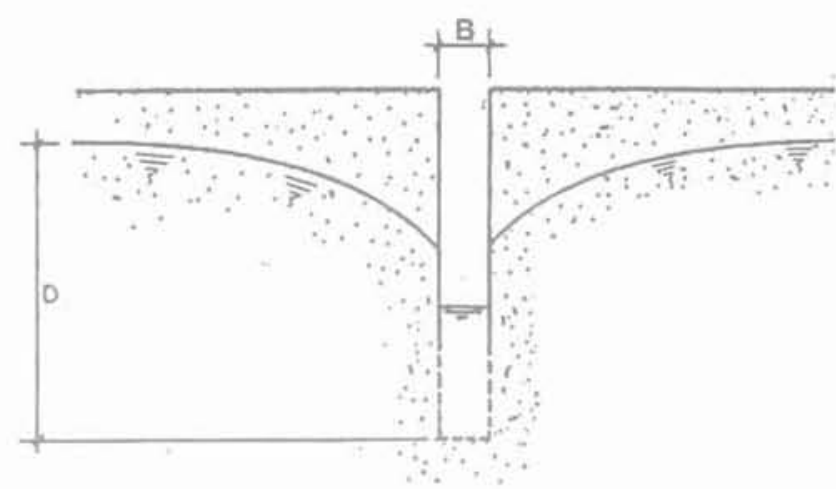

मंG. 8 Pompage en nappe libre avec substratum infiniment profond.

Pumping in an unconfined aquifer with bedrock at infinite depth

on obtient :

$$
q=\frac{\delta_{1}+\ln x}{n \delta_{1}+\ln (n x)}
$$

d'où :

$$
\ln x=\frac{q}{1-q}\left[(n-1) \delta_{1}+\ln (n)\right]
$$

En posant :

$$
\beta=\frac{q}{1-q}\left[(n-1) \delta_{1}+\ln (n)\right]
$$

on obtient finalement :

$$
\begin{aligned}
& x=e^{\beta} \\
& \alpha=\left[\frac{B e^{\beta}}{\pi D_{1}}\right]^{2}
\end{aligned}
$$

On a par ailleurs:

$$
\frac{k_{h}}{k_{i}}=\frac{\delta_{i}+\ln \left(\frac{\pi D_{i}}{B} \sqrt{\alpha}\right)}{\delta_{i}+\ln \left(\frac{\pi D_{i}}{B}\right)}
$$

ou directement:

$$
k_{h}=\frac{Q}{2 \pi h D_{i}}\left[\frac{D_{i}}{10 H_{0}}+\ln \frac{\pi D_{i}}{B} \sqrt{\alpha}\right]
$$

\section{- Analogie avec la nappe en charge}

En considérant toujours le système réel et son symétrique par rapport au niveau statique initial de la nappe, on pourra utiliser directement les résultats de la nappe en charge à savoir :

$\frac{D}{H_{o}}<0,3$; les formules (18) et (19) du puits sans piézomètres ou (20 bis) et (21) du puits avec piézomètres.

$\frac{D}{H_{o}}>0,3$; les formules (25), (26) et (27) du puits avec

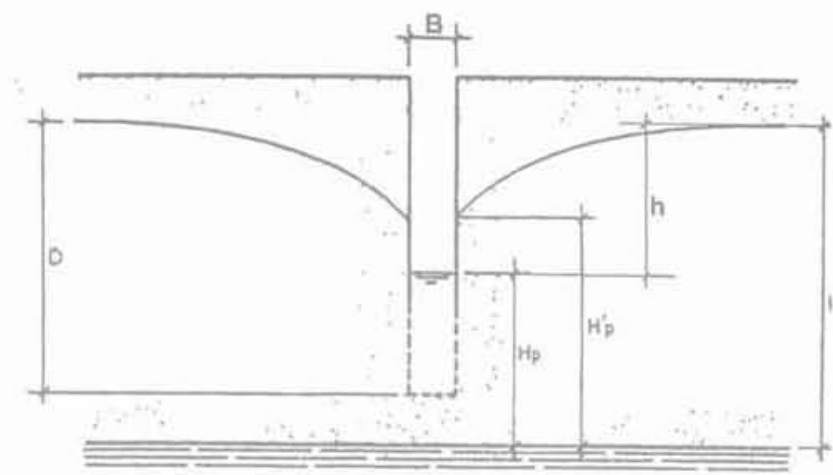

मG. 9 Puits imparfait dans une nappe libre avec substratum à distance finie.

Unperfect well in an unconfined aquifer with a bedrock at a finite depth.

\section{Recommandations pratiques concernant les dispositions expérimentales}

4.1

\section{Essais dans le puits en l'absence de piézomètres}

Il est extrêmement rare que l'on procède à un essai de pompage dans un puits qui ne soit pas assorti de piézomètres mais, bien que peu probable, ce cas peut se produire.

Comme nous l'avons indiqué précédemment, en l'absence de piézomètres il faut réaliser le forage du puits en deux ou trois phases, chaque phase faisant l'objet d'un pompage. On interprétera alors l'essai comme un essai Lefranc selon les méthodes précédemment exposées [5].

Comme les forages de puits sont réalisés en diamètres nettement supérieurs à ceux utilisés pour les essais Lefranc ou pour les piézomètres et qu'ils doivent obligatoirement être équipés d’un tube crépiné conformément à la norme NFP-94.130, il est recommandé d'utiliser ce tube crèpiné même pour les pompages intermédiaires.

\section{Datil}

\section{Nappe en charge}

La paroi du puits à travers l'éponte supérieure devra être étanche (tube de forage sans sabot débordant et cimentation si nécessaire) et le forage sera poursuivi en tubant au-dessous de cette éponte, aucune boue, même biodégradable, ne devant être utilisée (Fig. 10).

A la profondeur désirée, on arrêtera le forage et on mettra en place le tube crépiné et le matériau filtrant (Fig. 10-1). On remontera ensuite le tube de forage jusqu'à l'éponte supérieure et on réalisera un bouchon d'argile (Fig. 10-2).

On procédera ensuite au premier pompage, puis on retirera le tube crépiné et on approfondira le forage en 


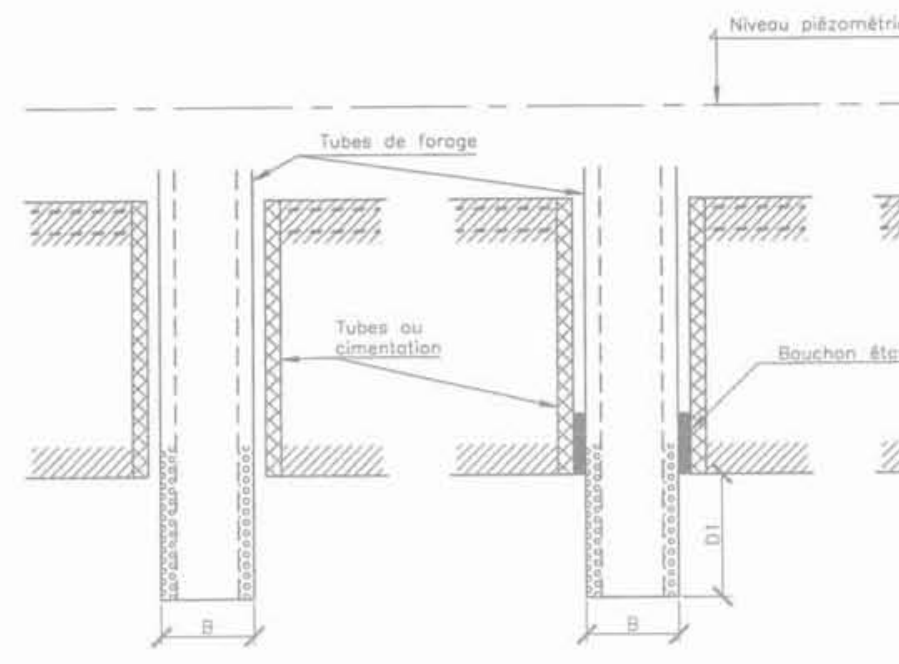

(1)

(2)
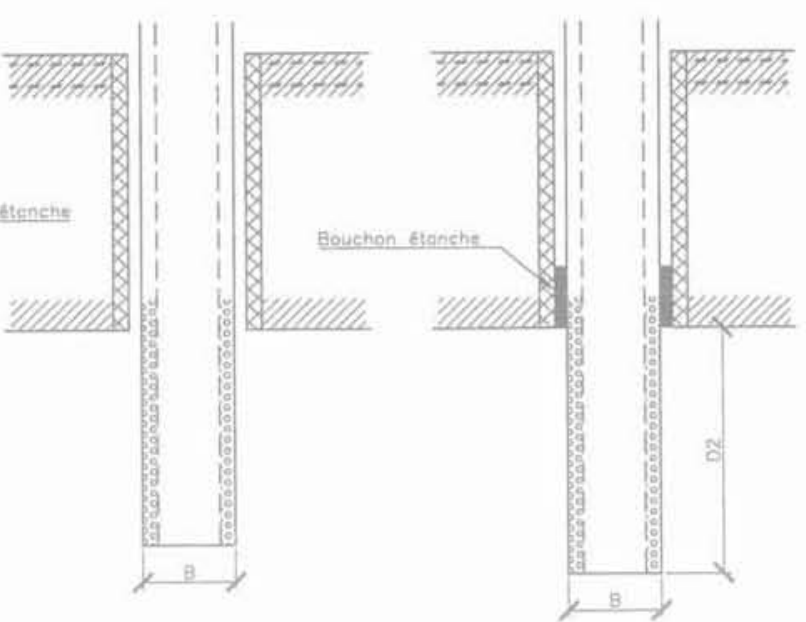

(3)

(4)

FG. 10 Réalisation des cavités à la base d'un puits dans une nappe en charge.

Creating a cavity at the bottom of a well in a confined aquifer.

détruisant évidemment le premier bouchon étanche (Fig. 10-3), Puis, on remettra en place le tube crépiné et on réalisera un autre bouchon filtrant pour procéder au deuxième essai (Fig. 10-4).

Les deux essais devront être précédés d'un développement selon les prescriptions de la norme NFP94.130.

\section{(a)}

\section{Nappe libre}

Le forage doit être tubé (tube lisse à sabot non débordant), à l'exclusion de toute utilisation de boue même biodégradable.

Lorsque le forage proprement dit aura atteint la cote désirée pour la première phase, on mettra en place le tube crépiné et on introduira un matériau filtrant dans l'espace annulaire entre les deux tubes. On remontera ensuite le tube de forage d'une hauteur $\mathrm{D}_{1}$, on développera le puits et on procédera au premier pompage.

Pour faire varier la hauteur de la cavité, la méthode la plus rapide consiste à opérer vers le haut. Dans ce cas, il suffit de rajouter du matériau filtrant et de remonter une nouvelle fois le tube de forage jusqu'à une hauteur $\mathrm{D}_{2}$.

Une fois les deux pompages terminés, on extraira le tube crépiné et on reprendra le forage du puits (Fig. 11).

\section{2}

\section{Utilisation de piézomètres}

En général, les puits pour essais de pompages réalisés selon la norme NFP-94.130 sont assortis d'au moins trois piézomètres, ce qui permet, s'ils sont convenablement espacés, de s'affranchir de la variation de la fiche du puits qui représente, il faut bien le reconnaitre, une sujétion très lourde.

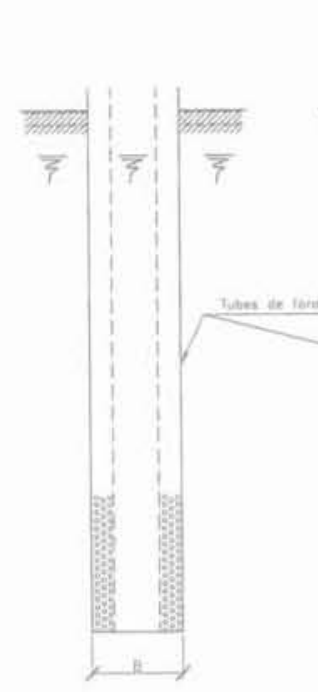

(1)

FIG. 11

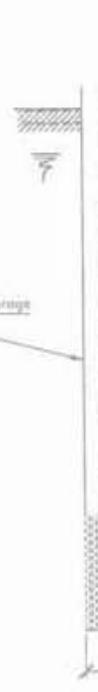

(3)

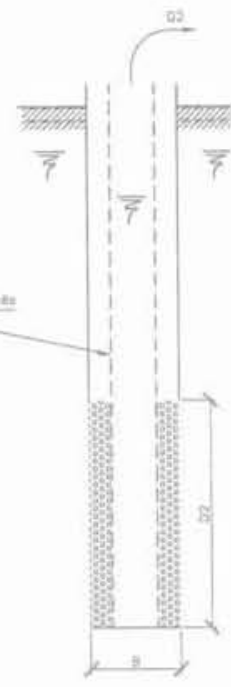

(2)
Réalisation des cavités à la base d'un puits en nappe libre.

Creating a cavity at the bottom of a well in an unconfined aquifer.

\section{6}

\section{Nappe en charge}

Dans le cas d'une nappe en charge, ces piézomètres doivent pénétrer dans l'aquifère sur une longueur d'environ un mètre, la prise de pression étant isolée hydrauliquement du reste du forage par un bouchon d'argile et une injection de bentonite-ciment (Fig. 12). 


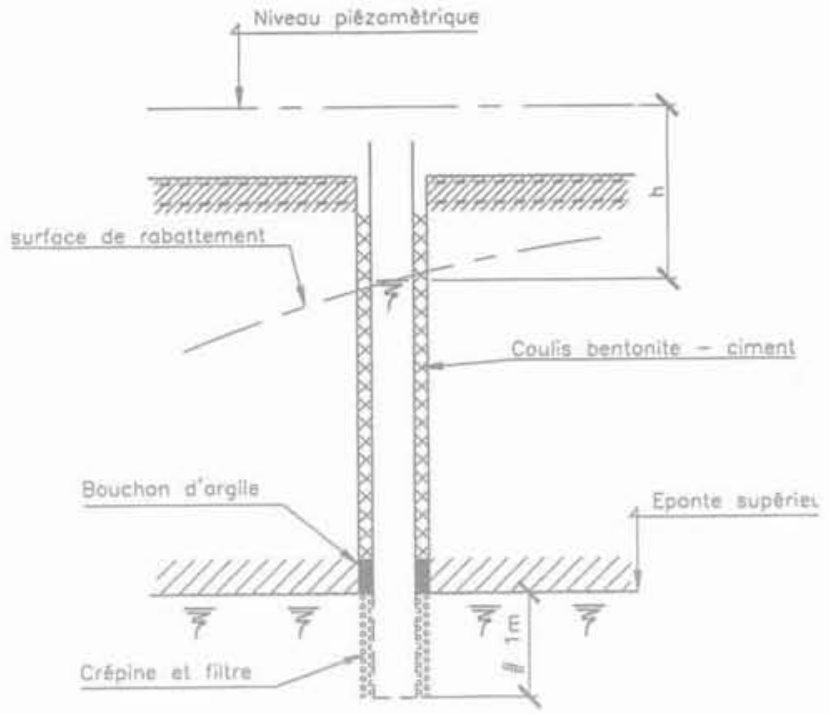

FG. 12 Piézométre en nappe captive. Piezometer in a confined aquifer.

La faible pénétration de la prise de pression dans l'aquifère, au-dessous de l'éponte supérieure, a pour but de saisir la variation de charge le plus près possible de l'axe de symétrie du dispositif fictif constitué par le dispositif réel et son image dans le miroir constitué par la base de l'éponte supérieure.

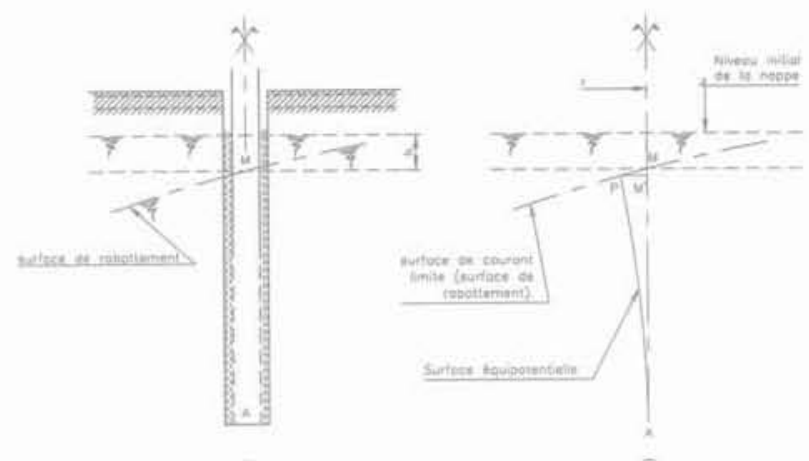

(9)

(b)

FG. 13 Piézomètre en nappe libre pour un puits crépiné sur toute sa hauteur. Piezometer in an unconfined aquifer for a well with an all length slotted casing.

\section{Nappe libre}

Dans le cas d'une nappe libre, si le puits est crépiné sur toute sa hauteur, on pourra crépiner les piézomètres également sur toute leur hauteur à condition que ceux-ci soient suffisamment éloignés du puits pour que la variation de la charge hydraulique au point $M$. intersection de l'axe du piézomètre et de la surface de

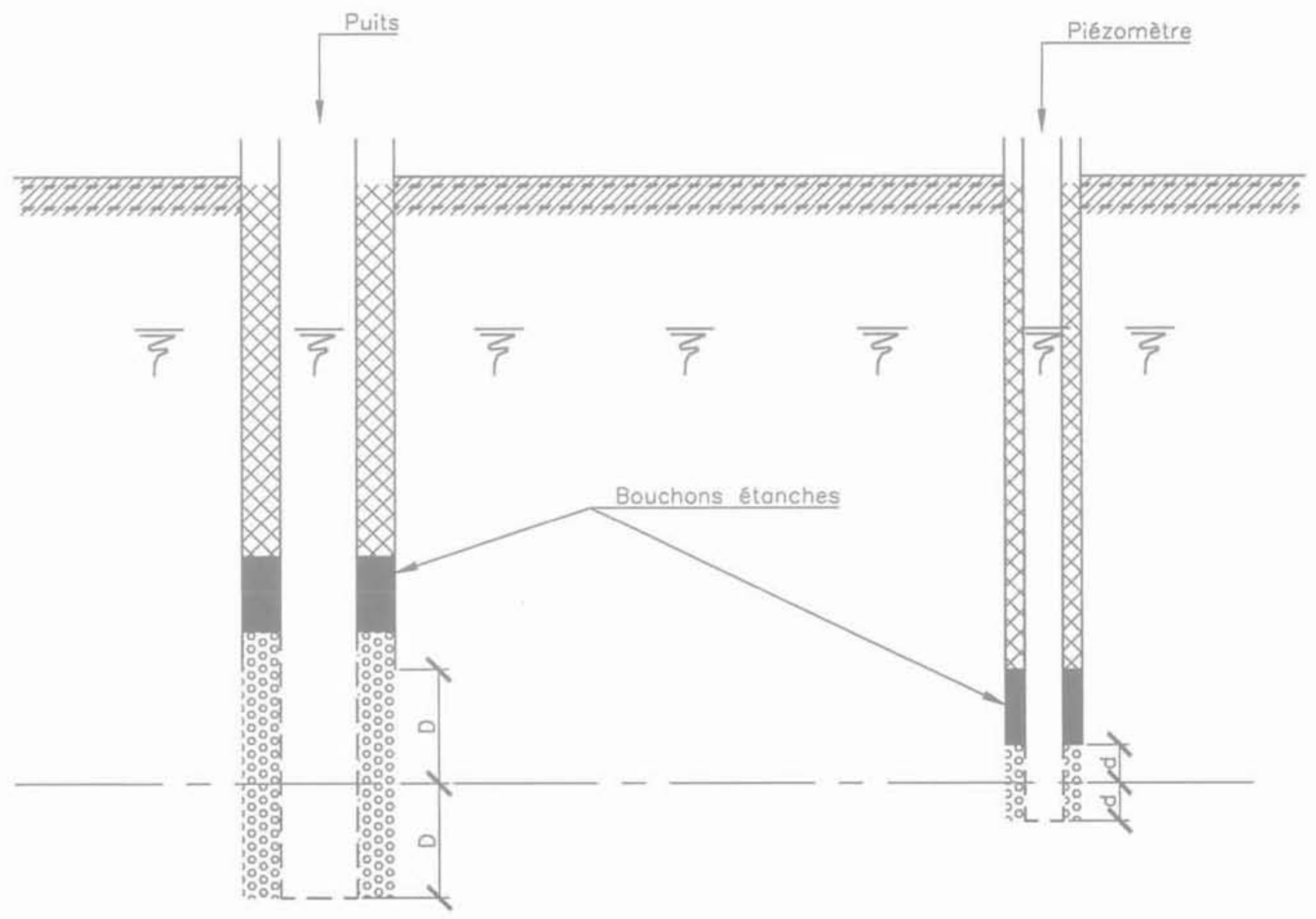

FG. 14 Piézomètre en nappe libre pour un puits crépiné à sa base sur une hauteur de 2D. Piezometer in a unconfined aquifer for a well with a $2 \mathrm{D}$ hight slotted casing from its base. 
rabattement, puisse être considérée comme égale à la hauteur de rabattement h mesurée dans le piézomètre c'est-à-dire si, sur la figure 13-b, le petit segment MM! est négligeable devant $h$, ce qui revient à admettre que la variation du potentiel entre l'équipotentielle (PA) passant par le pied du piézomètre et l'équípotentielle passant par le point $M$, est négligeable par rapport à la variation de charge $h$.

En revanche, si le puits n'est crépiné que sur une hauteur 2D à partir de sa base, les piézomètres devront être crépinés uniquement à leur base et symétriquement par rapport au plan horizontal passant par le milieu de la crépine du puits, cette partie crépinée devant être hydrauliquement isolée du reste du forage (Fig. 14).

\section{5}

\section{Récapitulation des procédures d'interprétation}

Les procédures d'interprétation que nous avons développées dans la présente étude sont récapitulées dans les quatre tableaux ci-après.
Nous donnons, par ailleurs en annexe, des tables numériques permettant la résolution des équations du type $q=f(n, x)$ qui interviennent dans la détermination de $\alpha$.

\section{6}

\section{Conclusion}

Au terme de cette étude, il apparait que l'essai de pompage est particulièrement bien adapté à l'étude de lanisotropie dans le cas, d'ailleurs très fréquent, du puits imparfait pourvu que l'on dispose d'au moins deux piézomètres, ce qui n'est pas un inconvénient puisque la norme française NFP-94.130 en impose un minimum de trois. Ce sont alors les distances des piézomètres au puits qui réalisent ipso facto les variations dimensionnelles nécessaires, et un seul pompage sous débit constant est suffisant.

L'approche du coefficient d'anisotropie n'est donc finalement qu'une question d'interprétation un peu particulière des mesures, l'essai n'étant entravé par aucune contrainte particulière si ce n'est celle de réaliser de véritables piézomètres avec prises de pression

TABLAUI Puits imparfait - Nappe en charge.

\begin{tabular}{|c|c|c|}
\hline \multirow[t]{2}{*}{ Essai de pompage } & \multicolumn{2}{|c|}{ 1) Substratum etanche infiniment profond } \\
\hline & Puits isolé sans piézomètres & Puits avec piézomètres \\
\hline Données du problème & $\begin{array}{l}D_{1} \text { et } D_{2}=\text { fiches du puits dans l'aquifère } \\
B=\text { diamètre du puits } \\
Q_{1} \text { et } Q_{2}=\text { débits de pompage } \\
h_{1} \text { et } h_{2}=\text { rabattements correspondants }\end{array}$ & $\begin{array}{l}D=\text { fiche du puits dans l'aquifère } \\
B_{0}=\text { diarnètre du puits } \\
r_{1} \text { et } r_{2}=\text { distances des piézomètres à l'axe du puits } \\
B_{1}=2 r_{1} \text { et } B_{2}=2 r_{2} \\
Q=\text { débit de pompage } \\
h_{1} \text { et } h_{2}=\text { rabattements dans piézomètres à } r \text {, et } r_{2}\end{array}$ \\
\hline Calculs auxiliaires & $\begin{array}{l}\lambda_{1}=\frac{2 D_{1}}{B} \quad \lambda_{2}=\frac{2 D_{2}}{B} \\
\mathrm{n}=\frac{\lambda_{2}}{\lambda_{1}}=\frac{D_{2}}{D_{1}} \\
\mathrm{q}=\frac{h_{1} Q_{2}}{n h_{2} Q_{1}} \\
* \mathrm{k}=\frac{Q_{1} \text { Argsh } \lambda_{1}}{\pi \lambda_{1} h_{1} B}=\frac{Q_{2} \text { Argsh } \lambda_{2}}{\pi \lambda_{2} h_{2} B}\end{array}$ & $\begin{array}{l}\lambda_{1}=\frac{D}{r_{1}} \quad \text { et } \quad \lambda_{2}=\frac{D}{r_{2}} \\
\mathrm{n}=\frac{r_{2}}{r_{1}} \\
\mathrm{k}_{\mathrm{k}}=\frac{\text { QArgsh } \lambda_{1}}{2 \pi \lambda_{1} h_{1} r_{1}}=\frac{\text { Argsh } \lambda_{2}}{2 \pi \lambda_{2} h_{2} r_{2}}\end{array}$ \\
\hline Calcul de $\alpha$ et $k_{n}$ & $\begin{array}{l}\text { Résoudire numériquement } \\
\frac{\operatorname{Argsh} x}{\operatorname{Argsh}(n x)}=q \rightarrow x \\
\alpha=\left(\frac{x}{\lambda_{1}}\right)^{2}=\left(x \frac{B}{2 D_{i}}\right)^{2} \\
\frac{k_{h}}{k}=\frac{\operatorname{Argsh}\left(\lambda_{i} \sqrt{\alpha}\right)}{\operatorname{Argsh} \lambda_{i}} \\
k_{h}=\frac{Q_{i}}{2 \pi \lambda_{i} B} \operatorname{Argsh}\left(\lambda_{i} \sqrt{\alpha}\right) \\
i=1 \text { et } 2\end{array}$ & $\begin{array}{l}\text { Résoudre numériquement } \\
\frac{\operatorname{Argshx}}{\operatorname{Argsh}(n x)}=\frac{h_{2}}{h_{1}} \rightarrow \mathrm{x} \\
\alpha=\left(\frac{x}{\lambda_{2}}\right)^{2}=\left(x \frac{r_{2}}{D}\right)^{2} \\
\frac{\mathrm{k}_{\mathrm{h}}}{\mathrm{k}}=\frac{\operatorname{Argsh}\left(\lambda_{1} \sqrt{\alpha}\right)}{\operatorname{Argsh} \lambda_{1}} \\
k_{h}=\frac{Q}{2 \pi h_{1} D} \operatorname{Argsh}(\lambda, \sqrt{\alpha}) \\
\quad \mathrm{i}=1 \mathrm{et} 2\end{array}$ \\
\hline
\end{tabular}

* En régime transitoire on caiculera $k_{1}$ selon la procédure de la norme NFP.94.132 - Essai Lefrane. 


\begin{tabular}{|c|c|c|}
\hline \multirow[t]{2}{*}{ Essai de pompage } & \multicolumn{2}{|c|}{ 2) Les deux horizons étanches à distance finie } \\
\hline & Puits isolé sans piézomètres & Puits avec plézomètres: \\
\hline Données du problème & $\begin{array}{l}H=\text { épaisseur de l'aquifère } \\
D_{1} \text { et } D_{2}=\text { fiches du puits dans l'aquifère } \\
B=\text { diamètre đu puits } \\
Q_{1} \text { et } Q_{2}=\text { débits de pompage } \\
h_{1} \text { et } h_{2}=\text { rabattements correspondants }\end{array}$ & $\begin{array}{l}H=\text { épaisseur de l'aquifère } \\
D=\text { fiche du puits dans l'aquifère } \\
r_{3} r_{2} \text { et } r_{3}=\text { distances des piézomètres au puits } \\
Q=\text { débit de pompage } \\
h_{1}, h_{2} \text { et } h_{1}=\text { rabattements dans les plézomètres. } \\
\text { a } r_{1}, r_{2} \text { et } r_{3}\end{array}$ \\
\hline Calculs auxiliaires & $\begin{array}{c}\lambda_{1}=\frac{2 D_{1}}{B}, \lambda_{2}=\frac{2 D_{2}}{B}, \mu_{1}=\frac{D_{1}}{H}, \mu_{2}=\frac{D_{2}}{H} \\
\mathrm{n}=\frac{D_{2}}{D_{1}}=\frac{\lambda_{2}}{\lambda_{1}}=\frac{\mu_{2}}{\mu_{1}}\left(D_{2}>D_{1}\right) \\
q=\frac{h_{1} Q_{2}}{\pi h_{2} Q 1} \\
* \mathrm{k}=\frac{Q\left[2 \pi \mu_{1}+\operatorname{Argsh} \lambda_{1}\right]}{2 \pi h_{1} D_{i}}\end{array}$ & $\begin{array}{c}\lambda_{1}=\frac{D}{r_{1}} \lambda_{2}=\frac{D}{r_{2}} \mathrm{n}=\frac{r_{2}}{r_{1}} \mu=\frac{D}{H} \\
\mu<0,3 \rightarrow \mathrm{k}=\frac{\mathrm{Q}}{2 \pi D h}\left[2 \pi \mu+\text { Argsh } \lambda_{1-1}\right] \text { avec } \mathrm{i}=1 \mathrm{et} 2 \\
\mu>0,3 \rightarrow \Phi\left(\mathrm{r}_{1}\right)=\frac{1}{\mu} \operatorname{Argsh} \lambda_{1}-\operatorname{Argsh} \frac{\lambda_{1}}{\mu} \mathrm{i}=1,2,3 \\
\cdots \mathrm{k}=\frac{Q}{2 \pi\left(h_{1}-h_{2}\right) H}\left[\ln \frac{r_{2}}{r_{1}}+\Phi\left(r_{2}\right)-\Phi\left(r_{1}\right)\right]\end{array}$ \\
\hline Calcul de $\alpha$ et $k_{h}$ & $\begin{array}{c}\text { Bésoudre numériquement } \\
\mathrm{q}=\frac{2 \pi \mu_{1}+\operatorname{Argsh} x}{2 \pi n \mu_{1}+\operatorname{Argsh}(n x)} \\
\alpha=\left(\frac{x}{\lambda_{1}}\right)^{2} \\
\frac{k_{h}}{k}=\frac{2 \pi \mu_{i}+\operatorname{Argsh}\left(\lambda_{i} \sqrt{\alpha}\right)}{2 \pi \mu_{i}+\operatorname{Argsh} \lambda_{i}} \\
k_{h}=\frac{Q_{i}}{2 \pi h D_{i}}\left[2 \pi \mu_{i}+\operatorname{Argsh}\left(\lambda_{i} \sqrt{\alpha}\right)\right] \\
\text { aveci }=1 \text { et } 2\end{array}$ & 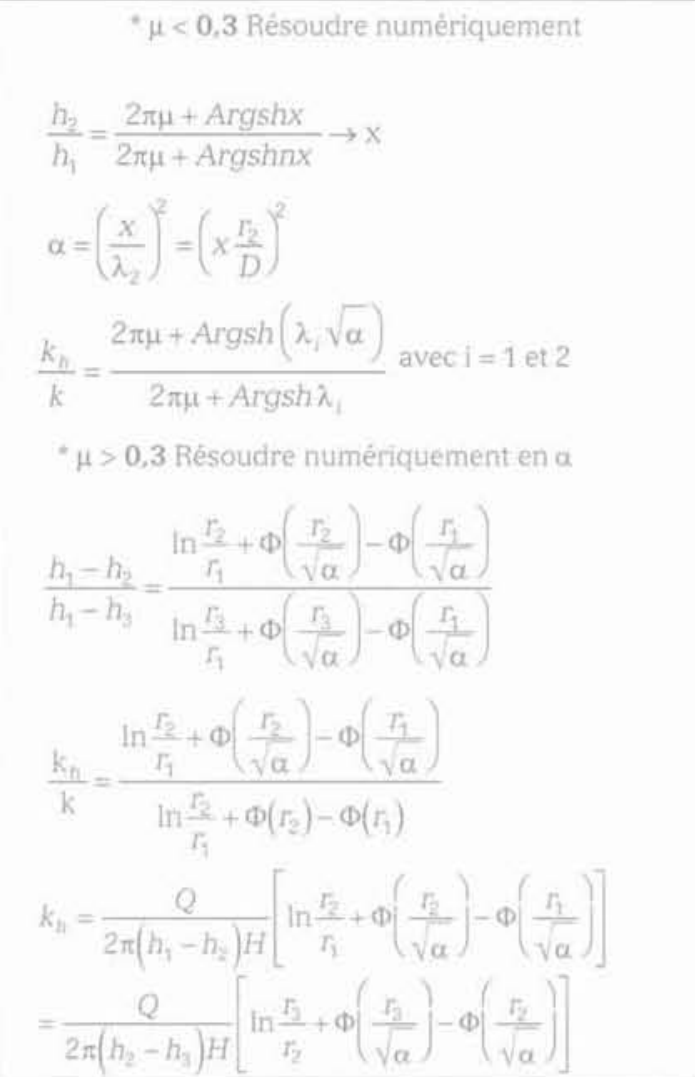 \\
\hline
\end{tabular}

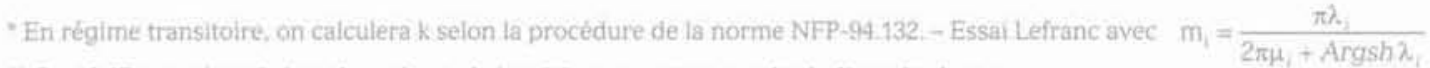

* On vêrifiera qu' on obtient des valeurs de $k$ voisines en permutant les indices des hi et $r_{\text {. }}$ 
TABLEAU III Puits imparfait - Nappe libre.

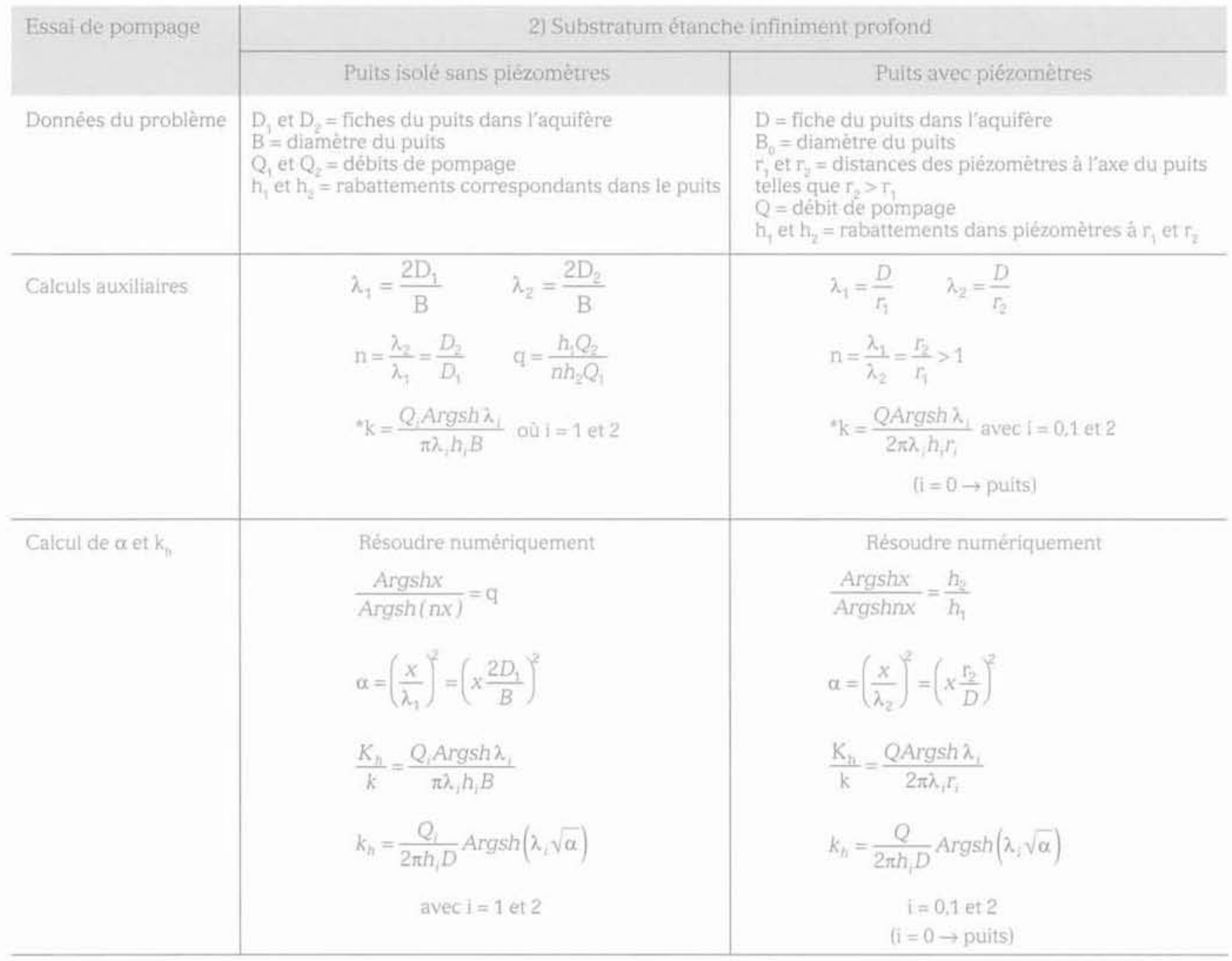

"En régime transitoire on calculera K selon la procédure de la norme NFP-94.132. 


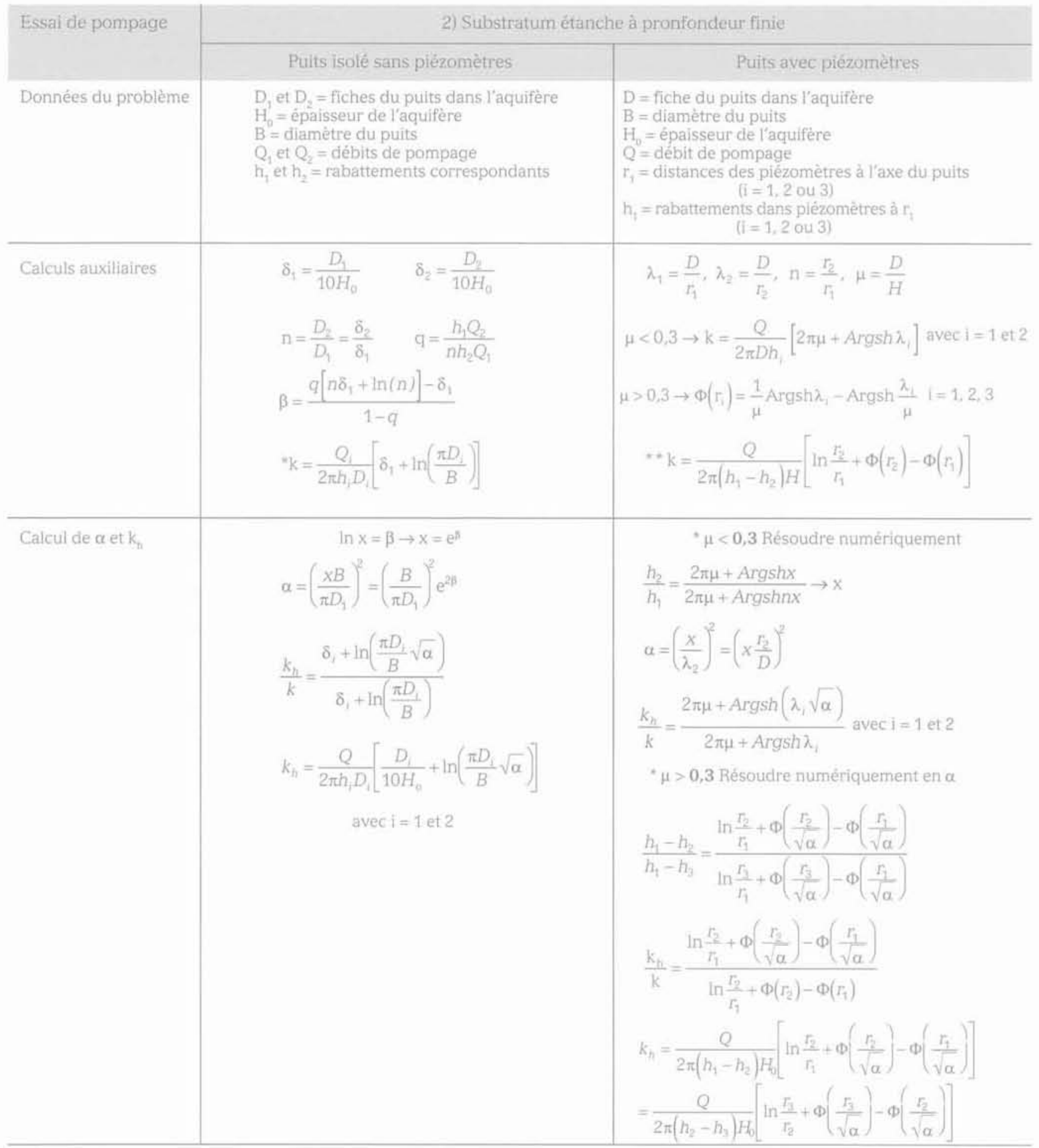

* En régime transitoire, on calculera $k$ selon la méthode de la norme NFP-94.132, avec $m_{3}=\frac{\pi \lambda_{i}}{2 \pi \mu_{i}+A r g s h \lambda_{1}}$

"On vérifiera qu'on obtient des valeurs de $k$ très voisines en permutant les indices des h et $r_{\text {, }}$ 
localisées à la base des tubes piézométriques, hydrauliquement isolées du reste de la nappe, et disposées à une profondeur convenablement choisie. Aucune interprétation fiable n'est possible avec des piézomètres crépinés sur toute leur hauteur, sauf si ceux-ci sont suffisamment éloignés du puits et si le puits lui-même est entièrement crépiné.

Dans le cas d'un puits parfait, l'essai ne permet de déterminer que le coefficient de perméabilité horizontale. Pour obtenir le coefficient d'anisotropie, il faut se ramener au cas du puits imparfait en arrêtant le forage du puits avant qu'il n'atteigne le substratum et en procédant, à ce niveau intermédiaire, à un pompage spécifique.

Dans le cas extrêmement rare où aucun piézomètre n'est prévu, il faut réaliser le puits en deux phases au moins avec pompage pour chacune d'elles, ce qui nous ramène au cas de l'essai Lefranc.

L'intérêt de l'utilisation d'un essai de pompage pour déterminer le coefficient d'anisotropie réside, comme nous l'avons dit en préambule, dans le fait que les résultats obtenus correspondent à une masse de sol importante et se trouvent, alors beaucoup plus représentatifs, à l'échelle d'un projet, que ceux provenant d'essais Lefranc.

Nous estimons donc que tout essai de pompage devrait être interprété selon les méthodes de la norme complétées par la méthode « anisotropique » que nous avons présentée, ce qui valoriserait considérablement les études hydrogéologiques.

\section{Bibliographie}

[1] Cambefort H. - Introduction à la géotechnique. Paris, Eyrolles, 1971.

[2] Cassan M. - « Filtration dans les cavités souterraines $x$. Annales de I'TTBTP, avrilmal 1979.

[3] Cassan M. - Les essais d'eau dans la reconnaissance des sols. Paris, Eyrolles, 1980.
[4] Cassan M. - Aide-mémoire d'hydraulique souterraine. Paris. Presses des Ponts et Chaussées, 1994, $2^{\prime}$ éd

[5] Cassan M. - $\alpha$ Application des essais Lefranc à l'évaluation du coefficient d'anisotropie hydraulique des sols aquifères $\gg$. Revue française de géotechnique, $\mathrm{n}^{\circ}$ 90, $1^{\text {tr }}$ trimestre 2000 .
[6] Mandel J, - $\alpha$ Note sur le calcul des filtrations . . Annales des Ponts et Chaussées, 1939.

[7] Schneebell G. - « La mesure in situ de la perméabilitẻ d'un terrain \%. Congrès de la Sté Hydrotechnique de France-Alger, 1954.

[8] Schneebeli G, - Hydraulique souterraine. Eyrolles, 1966. 


\section{Annexe}

Table numérique 1 $\quad y(x, n)=\frac{\operatorname{Argsh} x}{\operatorname{Argsh}(n x)}$

\begin{tabular}{|c|c|c|c|c|c|c|c|c|}
\hline & 1,5 & 2 & 2.5 & 3 & 3,5 & 4 & 4,5 & 5 \\
\hline 1,0 & 0,7376973 & 0,6105236 & 0,5350637 & 0,4846849 & 0,4483718 & 0,4207611 & 0,3689293 & 0,3811447 \\
\hline 1.5 & 0,7707364 & 0,6570241 & 0,5879090 & 0,5407765 & 0,5061860 & 0,4794819 & 0,4580915 & 0,4404713 \\
\hline 2.0 & 0,7938840 & 0,6891807 & 0,6242914 & 0,5793592 & 0,5459794 & 0.5199531 & 0,4989333 & 0,4814970 \\
\hline 2,5 & 0,8105556 & 0,7123352 & 0,6505477 & 0,6072819 & 0,5748594 & 0,5494025 & 0,5287239 & 0,5114875 \\
\hline 3,0 & 0,8230694 & 0,7297781 & 0,6704036 & 0,6284713 & 0,5968416 & 0,5718771 & 0,5515114 & 0,5344746 \\
\hline 3,5 & 0,8328179 & 0,7434307 & 0,6860075 & 0,6451792 & 0,6142234 & 0,5896906 & 0,5696100 & 0,5527644 \\
\hline 4,0 & 0,8406491 & 0,7544511 & 0,6986514 & 0,6587591 & 0,6283866 & 0,6042363 & 0,5844150 & 0,5677491 \\
\hline 4,5 & 0.8470996 & 0,7635702 & 0,7091505 & 0,6700667 & 0,5402062 & 0,6163976 & 0,5968127 & 0,5803145 \\
\hline 5.0 & 0,8525227 & $0, \pi 712696$ & 0,7180433 & 0,6796678 & 0,6502621 & 0,6267613 & 0,6073926 & 0,5910502 \\
\hline 5,5 & 0,8571598 & 0,7778790 & 0,7256990 & 0,6879517 & 0,6589538 & 0.6357321 & 0,6165619 & 0,6003645 \\
\hline 6,0 & 0,8611813 & 0,7836314 & 0,7323796 & 0,6951949 & 0,6665657 & 0,6435989 & 0,6246116 & 0,6085494 \\
\hline 6,5 & 0,8647108 & 0,7886969 & 0,7382762 & 0,7015997 & 0,6733063 & 0,6505734 & 0.6137555 & 0,6158195 \\
\hline 7,0 & 0,8678404 & 0,7932019 & 0,7435319 & 0,7073178 & 0.6793320 & 0,6568150 & 0,6381545 & 0,6223365 \\
\hline 7,5 & 0,8706399 & 0,7972430 & 0,7482557 & 0,7124650 & 0,6847627 & 0,6624458 & 0,6439321 & 0,6282249 \\
\hline 8,0 & 0,8731634 & 0,8008952 & 0,7525327 & 0,7171318 & 0,6896919 & 0,6675613 & 0,6491849 & 0,6335820 \\
\hline 8,5 & 0,8754535 & 0,8042175 & 0,7564300 & 0.7213897 & 0,6941939 & 0,6722373 & 0,6539898 & 0,6384853 \\
\hline 9,0 & 0,8775443 & 0,8072573 & 0,7600014 & 0,7252963 & 0,6983283 & 0,6765348 & 0,6584087 & 0,6429971 \\
\hline 9,5 & 0,8794631 & 0,8100529 & 0,7632908 & 0,7288983 & 0,7021437 & 0,6805036 & 0,6624921 & 0,6471686 \\
\hline 10,0 & 0,8812324 & 0,8126358 & 0,7663341 & 0,7322343 & 0,7056802 & 0,6841848 & 0,6662817 & 0,6510417 \\
\hline 15,0 & 0,8936610 & 0,8309208 & 0,7879967 & 0,7560794 & 0,7310420 & 0,7106555 & 0,6935939 & 0,6790110 \\
\hline 20,0 & 0,9010609 & 0,8419329 & 0,8011482 & 0,7706437 & 0,7466072 & 0,7269653 & 0,7104781 & 0,6963506 \\
\hline 25,0 & 0,9061430 & 0,8495534 & 0,8102976 & 0,7808168 & 0,7575141 & 0,7384239 & 0,7223663 & 0,7085827 \\
\hline 30,0 & 0,9099296 & 0,8552626 & 0,8171790 & 0,7884906 & 0,7657607 & 0,7471044 & 0,7313868 & 0,7178770 \\
\hline 35,0 & 0,9129035 & 0,8597659 & 0,8226231 & 0,7945756 & 0,7723118 & 0,7540103 & 0,7385725 & 0,7252888 \\
\hline 40,0 & 0,9153268 & 0,8634481 & 0,8270856 & 0,7995726 & 0,7776995 & 0,7596969 & 0,7444954 & 0,7314035 \\
\hline 45,0 & 0,9173559 & 0,8665403 & 0,8308407 & 0,8037840 & 0,7822457 & 0,7645001 & 0,7495025 & 0,7365767 \\
\hline 50.0 & 0,9190909 & 0,8691907 & 0,8340649 & 0,8074048 & 0,7861585 & 0,7686377 & 0,7538189 & 0,7410390 \\
\hline 55,0 & 0,9205992 & 0,8714997 & 0,8368780 & 0.8105674 & 0,7895793 & 0,7722578 & 0,7575979 & 0,7449479 \\
\hline 60,0 & 0,9219281 & 0,8735379 & 0,8393644 & 0,8133657 & 0,7926084 & 0,7754654 & 0,7609483 & 0,7484152 \\
\hline 65,0 & 0,9231120 & 0,8753568 & 0,8415859 & 0,8158680 & 0,7953192 & 0,7783377 & 0,7639498 & 0,7515227 \\
\hline 70,0 & 0,9241768 & 0,8769950 & 0,8435888 & 0,8181259 & 0,7977667 & 0,7809324 & 0,7666625 & 0.7543324 \\
\hline 75,0 & 0,9251419 & 0,8784820 & 0.8454085 & 0,8201789 & 0,7999933 & 0,7832941 & 0,7691326 & 0,7568917 \\
\hline 80,0 & 0,9260228 & 0,8798408 & 0,8470730 & 0.8220578 & 0,8020324 & 0,7854579 & 0,7713965 & 0,7592381 \\
\hline 85.0 & 0,9268317 & 0,8810900 & 0,8486042 & 0,8237875 & 0,8039104 & 0,7874514 & 0.7734831 & 0,7614013 \\
\hline 90,0 & 0,9275783 & 0.8822442 & 0,8500201 & 0,8253879 & 0,8056487 & 0,7892974 & 0,7754159 & 0,7634057 \\
\hline 95,0 & 0,9282707 & 0,8833156 & 0,8513354 & 0,8268752 & 0,8072649 & 0,7910144 & 0,7772140 & 0.7652709 \\
\hline 100,0 & 0,9289155 & 0,8843141 & 0.8525620 & 0,8282630 & 0,8087736 & 0,7926176 & 0,7788935 & 0,7670135 \\
\hline
\end{tabular}


Table numérique 1 (suite)

\begin{tabular}{|c|c|c|c|c|c|c|c|c|}
\hline & 6 & 7 & 8 & 9 & 10 & 12 & 14 & 16 \\
\hline 1,0 & 0,3537125 & 0,3333333 & 0,3174437 & 0,3046016 & 0,2939653 & 0,2771802 & 0,2644006 & 0.2542391 \\
\hline 1,5 & 0,4129208 & 0,3921393 & 0,3757371 & 0,3623563 & 0,3511627 & 0,3333333 & 0,3196061 & 0.3085940 \\
\hline 2,0 & 0,4540041 & 0,4330719 & 0,4164280 & 0,4027675 & 0,3912817 & 0,3728749 & 0.3586075 & 0,3471006 \\
\hline 2.5 & 0,4841513 & 0,4632046 & 0,4464641 & 0,4326671 & 0,4210259 & 0,4022914 & 0,3877025 & 0,3758928 \\
\hline 3,0 & 0,5073380 & 0,4864451 & 0,4696844 & 0,4558279 & 0,4441060 & 0,4251827 & 0,4103957 & 0,3983928 \\
\hline 3,5 & 0,5258417 & 0,5050362 & 0,4882964 & 0,4744236 & 0,4626641 & 0,4436332 & 0,4287219 & 0,4165917 \\
\hline 4,0 & 0,5410408 & 0,5203388 & 0,5036423 & 0,4897783 & 0,4780067 & 0,4589183 & 0,4439287 & 0,4317133 \\
\hline 4,5 & 0,5538147 & 0,5332227 & 0,5165818 & 0,5027413 & 0,4909735 & 0,4718593 & 0,4568218 & 0,4445491 \\
\hline 5,0 & 0,5647501 & 0,5442697 & 0,5276910 & 0,5138828 & 0,5021287 & 0,4830097 & 0,4679445 & 0,4556338 \\
\hline 5,5 & 0.5742543 & 0,5538843 & 0,5373707 & 0,5236002 & 0,5118662 & 0,4927562 & 0,4776775 & 0,4653421 \\
\hline 6,0 & 0,5826191 & 0,5623568 & 0,5459094 & 0,5321795 & 0,5204696 & 0,5013781 & 0,4862959 & 0,4739456 \\
\hline 6.5 & 0,5900596 & 0,5699016 & 0,5535201 & 0,5398323 & 0,5281490 & 0.5090826 & 0,4940039 & 0,4816459 \\
\hline 7,0 & 0,5967379 & 0,5766804 & 0,5603638 & 0,5467188 & 0,5350637 & 0,5160267 & 0,5009569 & 0,4885964 \\
\hline 7,5 & 0,6027790 & 0,5828181 & 0,5665651 & 0,5529629 & 0,5413368 & 0,5223323 & 0,5072750 & 0,4949161 \\
\hline 8,0 & 0,6082810 & 0,5884129 & 0,5722218 & 0,5586619 & 0,5470652 & 0,5280952 & 0,5130532 & 0,5006990 \\
\hline 8.5 & 0,6133218 & 0.5935427 & 0,5774117 & 0,5638936 & 0,5523263 & 0,5333920 & 0,5183674 & 0,5060202 \\
\hline 9,0 & 0,6179645 & 0,5982708 & 0.5821981 & 0,5687209 & 0,5571829 & 0,5382851 & 0,5232793 & 0,5109408 \\
\hline 9,5 & 0,6222606 & 0,6026489 & 0,5866326 & 0,5731954 & 0,5616864 & 0,5428255 & 0,5278395 & 0,5155112 \\
\hline 10,0 & 0,6262527 & 0,6067197 & 0,5907581 & 0,5773599 & 0,5658794 & 0,5470555 & 0,5320902 & 0,5197731 \\
\hline 15,0 & 0,6551733 & 0,6362865 & 0,6207845 & 0,6077245 & 0,5964989 & 0,5780228 & 0,5632715 & 0.5510888 \\
\hline 20.0 & 0,6731865 & 0,6547708 & 0,6396138 & 0,6268151 & 0,6157927 & 0,5976076 & 0,5830498 & 0.5710006 \\
\hline 25,0 & 0,6859334 & 0,6678836 & 0,6529987 & 0,6404094 & 0,6295522 & 0,6116092 & 0,5972178 & 0,5852878 \\
\hline 30,0 & 0,6956413 & 0.6778884 & 0,6632266 & 0,6508106 & 0,6400915 & 0,6223537 & 0,6081059 & 0,5962810 \\
\hline 35,0 & 0.7033967 & 0,6859925 & 0,6714190 & 0,6591502 & 0,6485492 & 0.6309884 & 0.6168662 & 0,6051343 \\
\hline 40,0 & 0,7098043 & 0,6925133 & 0.6782021 & 0,6660608 & 0,6555627 & 0,6381571 & 0,6241461 & 0,6124973 \\
\hline 45,0 & 0,7152319 & 0,6981270 & 0,6839580 & 0,6719291 & 0,6615218 & 0,6442542 & 0,6303427 & 0,6187687 \\
\hline 50,0 & 0,7199185 & 0,7029785 & 0,6889359 & 0,6770071 & 0,6666810 & 0,6495374 & 0,6357158 & 0,6242099 \\
\hline 55,0 & 0,7240277 & 0,7072353 & 0.6933062 & 0,6814676 & 0,6712150 & 0,6541836 & 0,6404440 & 0,6290003 \\
\hline 60,0 & 0,7276755 & 0,7110165 & 0,6971905 & 0,6854338 & 0,6752481 & 0,6583194 & 0,6446549 & 0,6332685 \\
\hline 65,0 & 0,7309472 & 0,7144100 & 0,7006780 & 0,6889963 & 0,6788720 & 0,6620377 & 0,6484425 & 0,6371093 \\
\hline 70,0 & 0,7339072 & 0,7174817 & 0,7038362 & 0,6922237 & 0,6821560 & 0,6654091 & 0,6518782 & 0,6405944 \\
\hline 75.0 & 0,7366051 & 0,7202827 & 0,7067172 & 0,6951688 & 0,6851536 & 0,6684880 & 0,6550171 & 0,6437794 \\
\hline 80,0 & 0,7390799 & 0,7228531 & 0,7093620 & 0,6978733 & 0,6879071 & 0,6713173 & 0,6579026 & 0,6467082 \\
\hline 85,0 & 0,7413626 & 0,7252251 & 0,7118034 & 0,7003705 & 0,6904501 & 0,6739315 & 0,6605995 & 0,6494160 \\
\hline 90,0 & 0,7434786 & 0.7274246 & 0.7140681 & 0.7026874 & 0,6928101 & 0,6763584 & 0,6630462 & 0,6519312 \\
\hline 95,0 & 0,7454486 & 0,7294730 & 0,7161777 & 0,7048464 & 0,6950097 & 0,6786211 & 0,6653560 & 0,6542774 \\
\hline 100,0 & 0,7472898 & 0,7313881 & 0,7181506 & 0,7068658 & 0,6970675 & 0,6807388 & 0,6675182 & 0.6564743 \\
\hline
\end{tabular}


Table numérique 2 $\quad \frac{k_{\mathrm{H}}}{k}=\frac{\operatorname{Argsh}(\lambda \sqrt{\alpha})}{\operatorname{Argsh} \lambda}$

\begin{tabular}{l|l|l|l|l|l|l|l|l|}
\hline$\alpha$ & $\lambda$ & 1,5 & 2 & 2,5 & 3 & 3,5 & 4 & 5 \\
\hline 5 & 1,61107 & 1,52621 & 1,47038 & 1,43089 & 1,40136 & 1,37836 & 1,34460 & 1,26773 \\
10 & 1,89229 & 1,76206 & 1,67840 & 1,61996 & 1,57664 & 1,54307 & 1,49404 & 1,38324 \\
15 & 2,05896 & 1,90108 & 1,80067 & 1,73095 & 1,67943 & 1,63961 & 1,58157 & 1,45083 \\
20 & 2,17783 & 2,00000 & 1,88759 & 1,80979 & 1,75243 & 1,70815 & 1,64370 & 1,49880 \\
25 & 2,27030 & 2,07686 & 1,95508 & 1,87100 & 1,80909 & 1,76134 & 1,69190 & 1,53600 \\
30 & 2,34598 & 2,13972 & 2,01026 & 1,92103 & 1,85540 & 1,80481 & 1,73130 & 1,56640 \\
35 & 2,41006 & 2,19290 & 2,05694 & 1,96334 & 1,89456 & 1,84157 & 1,76461 & 1,59210 \\
40 & 2,46561 & 2,23900 & 2,09738 & 2,00000 & 1,92848 & 1,87342 & 1,79346 & 1,61437 \\
45 & 2,51464 & 2,27967 & 2,13307 & 2,03234 & 1,95841 & 1,90151 & 1,81892 & 1,63401 \\
50 & 2,55853 & 2,31606 & 2,16500 & 2,06128 & 1,98519 & 1,92664 & 1,84169 & 1,65158 \\
55 & 2,59825 & 2,34900 & 2,19388 & 2,08746 & 2,00941 & 1,94938 & 1,82229 & 1,66747 \\
60 & 2,63452 & 2,37907 & 2,22026 & 2,11136 & 2,03153 & 1,97014 & 1,88110 & 1,68198 \\
65 & 2,66790 & 2,40674 & 2,24452 & 2,13335 & 2,05188 & 1,98923 & 1,89840 & 1,69533 \\
70 & 2,69881 & 2,43235 & 2,26699 & 2,15371 & 2,07072 & 2,00692 & 1,91442 & 1,70768 \\
75 & 2,72760 & 2,45621 & 2,28791 & 2,17266 & 2,08826 & 2,02338 & 1,92933 & 1,71919 \\
80 & 2,75453 & 2,47853 & 2,30748 & 2,19040 & 2,10466 & 2,03878 & 1,94328 & 1,72995 \\
85 & 2,77983 & 2,49949 & 2,32586 & 2,20706 & 2,12008 & 2,05324 & 1,95639 & 1,74006 \\
90 & 2,80369 & 2,51926 & 2,34320 & 2,22276 & 2,13461 & 2,06688 & 1,96874 & 1,74959 \\
95 & 2,82627 & 2,53796 & 2,35959 & 2,23762 & 2,14835 & 2,07978 & 1,98043 & 1,75861 \\
100,0 & 2,84768 & 2,55570 & 2,37515 & 2,25171 & 2,16140 & 2,09202 & 1,99152 & 1,76716 \\
\hline
\end{tabular}

